\title{
Arbor
}

\section{Ciencia y tecnología de materiales}

\author{
Isidoro Rasines
}

Arbor CLXVI, 653 (Mayo 2000), 83-125 pp.

Se indica cúando nacen los institutos del Área de Ciencia y Tecnología de Materiales y se expone cómo se evalúan los resultados de la labor que realizan. Se describe esta labor comenzando con la que llevan a cabo los cuatro institutos sectoriales en los ámbitos de las Ciencias de la Construcción, la Ciencia y la Tecnología de Polímeros, la Cerámica y el Vidrio, y la Metalurgia. A continuación se consigna la que realizan los cuatro institutos de Ciencias de Materiales en Aragón, Barcelona, Madrid y Sevilla; y la Unidad de Física de Materiales, en Bilbao. Al pasar revista a la situación actual estos centros se hace referencia a sus líneas de trabajo y proyectos en curso, y se mencionan en algún caso sus horizontes de futuro. También se indican en sendos anexos datos sobre recursos humanos y presupuestarios.

La science, aujourd'hui, cherchera una source d'inspiration au-dessus d'elle ou périra. S. Weil

\section{Antecedentes}

En el Consejo Superior de Investigaciones Científicas (abreviadamente el Consejo, o CSIC) el estudio de los materiales se encuadra en el área de Ciencia y Tecnología de Materiales, que tiene su origen ${ }^{1}$ en el programa movilizador iniciado en 1984 con el informe de una 
comisión constituida por los Profs. S. de Aza, A. Bello, J.M. López Sancho, J.M. Martínez Duart y, como Presidente, J.M. Serratosa.

Existían entonces en el Consejo cuatro institutos que se dedicaban al estudio de materiales interesantes para determinados sectores industriales: el Instituto Eduardo Torroja de Ciencias de la Construcción, que había nacido en 1934 con carácter privado como Instituto Técnico de la Construcción y Edificación, y se convertiría en centro propio del Consejo en 1946; el Instituto de Ciencia y Tecnología de Polímeros, creado al final de los años cuarenta en el seno del Instituto Alonso Barba de Química; el Instituto de Cerámica y Vidrio, que comienza en 1965; y el Centro Nacional de Investigaciones Metalúrgicas, procedente de la unificación en 1963 de tres institutos anteriores, el del Hierro y el Acero, el de la Soldadura y el de Metales no Férreos.

Además de estos cuatro institutos sectoriales, en 1984 había en el ámbito del CSIC otros de menor tamaño como el de Físico-Química Mineral o el de Química Inorgánica Elhúyar en Madrid, y grupos de investigadores en física de la materia condensada o en química molecular y química del estado sólido, que trabajaban en Barcelona, Sevilla y Zaragoza. Estos centros se integrarán, como proponía el programa movilizador mencionado, en los cuatro institutos que se crean para potenciar el área de materiales.

El Instituto de Ciencia de Materiales de Aragón, Centro mixto de la Universidad de Zaragoza y del CSIC, nace en 1985. Inician sus tareas algo más tarde, entre 1986 y 1987, el Instituto de Ciencia de Materiales de Barcelona, el Instituto de Ciencia de Materiales de Madrid, y el Instituto de Ciencia de Materiales de Sevilla. Este último, como Centro mixto de la Universidad de Sevilla y el CSIC. También como Centro mixto de otra Universidad, la del País Vasco, y el CSIC se ha creado recientemente, en 1998, una Unidad de Física de Materiales que ha quedado adscrita al área de CTM.

Los objetivos del programa movilizador de 1984 se han ido cumpliendo al correr del tiempo. Se trataba entonces de favorecer la implantación y el desarrollo de grupos de trabajo que lograran resultados reconocidos internacionalmente. Diez años más tarde se afirmaba en una publicación ${ }^{2}$ del Centro para el Desarrollo Tecnológico Industrial, del Ministerio de Industria, que «Los resultados obtenidos hasta ahora por el CSIC en cuanto a la investigación y el desarrollo de nuevos materiales, han sido muy buenos».

En la misma publicación ${ }^{2}$ se señalaba que los trabajos de los grupos del CSIC en el área de materiales «son considerados entre los de más alto nivel internacional» desde el punto de vista científico; y que «además 


\section{Ciencia y Tecnología de materiales}

de ser competitivos en Europa, estos grupos de investigación han logrado numerosos contactos con industrias». El elogio, merecido por buen número de grupos de trabajo del área, resulta algo exagerado si se aplica a todos.

Es claro que hay que lograr el mejor nivel alcanzable para el personal científico y que esto se viene intentando de modo perseverante. Así, la dotación de becas postdoctorales para científicos jóvenes que pueden perfeccionar su formación en laboratorios acreditados de otros países e importar, a la vuelta, nuevas técnicas y líneas de trabajo; la dotación, también, de más plazas de ingreso en plantilla, accesibles a los doctores contratados; o el nombramiento, para la casi totalidad de los institutos del área, de comités científicos asesores constituidos por expertos del máximo nivel, la mayoría de otros países, que examinan periódicamente la labor realizada y formulan sus recomendaciones y sugerencias.

Esto plantea la cuestión de los resultados, es decir, la cuestión de cómo evaluar el trabajo científico. Aunque cabe adelantar que la calidad media de la investigación desarrollada por los institutos del área es ahora aún más elevada que hace tres o cuatro años ${ }^{1}$, la temática de los proyectos que se desarrollan en los cuatro institutos de creación reciente continúa siendo en gran medida básica, fundamental, con escasa implantación en la industria.

\section{Resultados}

Los resultados que obtienen los científicos se publican en revistas especializadas de difusión internacional y son de dominio público; los logros de los tecnólogos se protegen con patentes, cuyo valor depende de la calidad y la cantidad de los productos que permitan fabricar. En cuanto a las revistas, se admite su evaluación, aunque no sin reservas, según un índice de impacto ${ }^{3}$ que se calcula cada año, dividiendo el número de veces que se han citado durante ese año los artículos publicados en la revista a lo largo de los dos años anteriores, por el número de artículos que ha publicado la revista durante el mismo periodo.

Por ejemplo, en 1997 el ISI registró 50.084 citas de los 1.830 trabajos publicados en la revista Nature durante 1995 y 1996. El índice de impacto de Nature en 1997 es el cociente de esas dos cantidades, 27,37, un número muy elevado si se compara con índices como 0,03 , que corresponde a la revista de materiales menos citada. 
Todavía rara vez logran publicar en Nature o en Science los investigadores españoles del área de CTM. En cambio todos los años aparecen algunos de sus artículos en revistas como Angewandte Chemie, Physical Review Letters, The Journal of the American Chemical Society $y$ Chemistry of Materials, con índices de impacto 8,56;6,14; 5,65 y 3,27 respectivamente.

\section{Los institutos del área de CTM, hoy}

A continuación se pasará revista a la situación actual de los institutos que integran el área de CTM del CSIC y se mencionarán en algún caso sus horizontes de futuro. También se incluirán datos sobre recursos humanos y presupuestarios en los anexos 1 y 2. Para dar idea del trabajo científico actual, se hará también una breve referencia a las líneas de trabajo y a los proyectos en curso. Mientras no se indique otra cosa, los datos proceden de la última memoria publicada por los centros, en la mayor parte de los casos la del año 1998.

Por razones de espacio, no se incluirán aquí los detalles que constan en esas memorias sobre comunicaciones a congresos, técnicas experimentales disponibles, propuestas de proyectos para experimentos en grandes instalaciones (radiación de sincrotrón, haces de neutrones)...Tampoco se comentarán las estancias del personal en otros centros, ni las de personas de otros centros en los institutos del área.

Hay también otros muchos detalles que no cabe incluir aquí porque no suelen aparecer en las memorias de los institutos, aunque algunos pueden confirmar la opinión antes citada ${ }^{2}$ sobre los grupos del área de CTM. Buen número de estos grupos tienen prestigio reconocido internacionalmente por el nivel de lo que publican y porque mantienen colaboraciones habituales con sus colegas de otros países. Por eso en buen número de casos son requeridos como árbitros por los directores de revistas de alto índice de impacto, para dar su opinión sobre la calidad de los manuscritos que reciben para publicar; y también por quienes administran los programas marco de la UE para evaluar proyectos.

\section{Ciencias de la Construcción}

Las etapas que ha vivido el IETCC desde su fundación, así como sus importantes aportaciones al sector de la construcción a lo largo de su historia, pueden encontrarse en un trabajo citado ${ }^{1}$. Ahora com- 


\section{Ciencia y Tecnología de materiales}

prende 4 departamentos de investigación y otras tantas unidades de servicio. Son los departamentos: Edificación y habitabilidad; Ingeniería estructural y Mecánica de materiales compuestos; Químico-física de materiales de construcción; y Síntesis, caracterización y estabilidad de materiales. Y las unidades de servicio: Asistencia científico-técnica; $\mathrm{Ca}$ lidad de la construcción; Documentación y Publicaciones; y Gerencia.

Durante los dos últimos años el Instituto se ha propuesto como objetivos: participar activamente en el Programa Marco Europeo, en los planes nacionales, y en los de comunidades autónomas; desarrollar contratos de investigación con entidades públicas y privadas; transferir tecnología al sector productivo por medio de varias actividades; y fomentar las relaciones con empresas, asociaciones profesionales y entidades de derecho público relacionadas con los sectores de la construcción, la edificación y los materiales de puesta en obra.

En 1997 el Instituto ha trabajado en 37 proyectos: 12, financiados por la UE; 13, por planes nacionales; 1, por el Ministerio de Fomento; 3, por el Centro de Desarrollo Tecnológico e Industrial; y 8, por comunidades autónomas. A la vez, durante los dos últimos años ha aumentado notablemente el número de convenios y contratos del Instituto, así como la elaboración de dictámenes, peritajes e informes técnicos realizados a petición de juzgados, audiencias, compañías de seguros y comunidades de propietarios. Se incluye a continuación una breve referencia a los objetivos que se proponen algunos de los proyectos financiados por la UE.

1) Confección de una base europea de datos fiables sobre propiedades térmicas (conductividad, resistencia) de materiales de construcción (metales, plásticos, madera, hormigón...) realizando, en caso necesario, las medidas oportunas.

2) Diseño y caracterización de materiales naturales, intermedios en la industria del corcho, para mejorar la calidad de las pantallas y elementos aisladores acústicos (prefabricados, o preparados in situ) utilizados en construcción.

3) Estudio de la interacción y posible alteración mineral de las barreras arcillosas en contacto con los cementos utilizados en almacenamientos subterráneos de residuos radiactivos de alta actividad como materiales de revestimiento y/o sellado, para definir el tipo de cemento que produce un $\mathrm{pH}$ compatible con la estabilidad de los minerales de la arcilla.

4) Mejoramiento de la resistencia a la corrosión de las armaduras de acero galvanizado en ambientes muy alcalinos como el del hormigón, a través de un tratamiento de pasivación por medio de un nuevo filmógeno orgánico. 
5) Desarrollo de una metodología de diseño de durabilidad de estructuras de hormigón, basada en características mensurables.

6) Determinación del comportamiento mecánico, térmico - a altas temperaturas - y microestructural, de ocho tipos de hormigones para altas y ultra altas prestaciones.

7) Estudio sobre regeneración de escombros para emplearlos en la construcción de carreteras e infraestructuras relacionadas.

8) Búsqueda de nuevos materiales útiles en construcción que quepa obtener por vitrificación y cristalización controladas de residuos de plantas incineradoras.

9) Definición del papel que desempeñan los contaminantes atmosféricos, en el deterioro de morteros hidráulicos tanto antiguos como modernos (mortero de cemento, de cal, de puzolana), con vistas a la restauración de edificios.

$\mathrm{Si}$ a lo anterior se suman las certificaciones y homologaciones de materiales de construcción y los informes de idoneidad técnica de materiales y sistemas constructivos, junto con los cursos y seminarios organizados, se concluye que el IETCC es, de todos los institutos del área, el que ha obtenido una mayor cantidad de ingresos por actividades propias, del orden de los 245 millones (cfr. anexo 2). Gracias a estos ingresos ha podido contratar a 37 personas y suplir así bajas por jubilación de personal que no ha cubierto la Administración Central, aunque esto haya supuesto la renuncia a renovar y ampliar la infraestructura instrumental del Instituto.

Los contratos de investigación suscritos se extienden también a otros países. No se limitaron a las acciones integradas, un total de 6, con Francia, Italia (dos acciones), Marruecos, Portugal, y el Reino Unido. Además, se suscribió un convenio para renovar el sistema de normalización de la construcción y los servicios de ingeniería y diseño en la República de Cuba; y, en el ámbito de nuestro país, un convenio también con el Ministerio de Fomento, sobre pliego de condiciones generales para obras de hormigón de aplicación en obras marítimas e instalaciones portuarias, así como otro convenio con la Junta de Andalucía para estudiar conjuntos y yacimientos arqueológicos en su contexto geomorfológico, biológico y climático.

Fruto de los proyectos de investigación y de las acciones integradas son los 42 artículos publicados en 1997 por el personal del Instituto, 23 de los cuales en revistas de índice de impacto comprendido entre 0,30 y 0,91 . Como novedad, cabe destacar entre esas publicaciones el empleo de la radiación de sincrotrón de rayos $\mathrm{X}$ para el estudio de fases de vida precaria que se detectan durante el fraguado de algunos 
cementos. Entre las publicaciones del personal del Instituto hay también contribuciones a monografías especializadas y a actas de diversos congresos. De otra parte, en 1997 se acabaron cuatro tesis doctorales.

Las interacciones del IETCC con su entorno social son abundantes e intensas. Comprenden primero los servicios de asistencia científicotécnica que presta a través de estudios de patología de construcciones, tanto en lo relativo a edificación de obras públicas o industriales, como a la rehabilitación y restauración de edificios histórico-artísticos. Durante 1997 se elaboraron 139 informes, 85 de los cuales fueron objeto de contrato.

El Instituto tiene encomendada legalmente la apreciación técnica del empleo de nuevos materiales, sistemas y procedimientos constructivos en edificación y obras públicas. Los criterios para esa apreciación se incluyen en los documentos de idoneidad técnica, DIT, de los que hay 96 vigentes y publicados, y unos 25 en desarrollo. De otra parte, el IETCC, que instituyó en España el primer sello de calidad de productos de la construcción, otorgó en 1997 el sello de conformidad CIE$T A N$, como marca de calidad especialmente para forjados de edificación, a 19 sociedades del sector de la construcción.

La colaboración del personal del Instituto en comisiones y grupos de trabajo que realizan tareas de normalización y certificación, ha alcanzado durante 1997 a 57 comités españoles y 37 internacionales. El IETCC, de otra parte, es la sede de la Asociación Científico-Técnica del Hormigón Estructural, ACHE. Además, para facilitar el intercambio de conocimientos entre la administración pública, los colegios profesionales y las empresas, el Instituto ha promovido la creación de la asociación AMIET, que viene a ser foro de relación con esos tres sectores y de ellos entre sí. En AMIET se dan cita, con el Ministerio de Fomento e Industria, la Agrupación de Fabricantes de Cemento, la Asociación Nacional de Empresas Fabricantes de Hormigón Preparado, la Asociación Nacional de Derivados del Cemento y la Confederación Nacional de la Construcción, así como los Colegios de Ingenieros de Caminos, de Arquitectos, y de Arquitectos técnicos.

A escala internacional, el IETCC está integrado y participa en las actividades de buen número de organismos internacionales, como la Red Europea de Institutos de Investigación de la Construcción, ENBRI; la Unión Internacional de Laboratorios de Ensayos e Investigación de Materiales y Estructuras, RILEM; la Unión Europea para la Aprobación Técnica en Construcción, UEATC; la Organización Europea para las Aprobaciones Técnicas, EOTA; el Comité Euro-internacional del Hormigón, FIB; el Consejo Internacional de la Construcción, CIB; y la 
Federación Internacional de Organizaciones de Evaluación Técnica, WFTAO. La Directora del Instituto ha desempeñado por cuarto año consecutivo la presidencia de la UEATC, y es Vicepresidente de la RILEM. Y dan idea de las relaciones con América tanto los 25 miembros correspondientes de la Red de Miembros del Instituto, como la participación del mismo Instituto en las Redes CYTEO-DURAR, sobre durabilidad de la armadura, y REHABILITAR, para rehabilitación de estructuras de hormigón.

Entre las directrices de futuro ${ }^{4}$ aprobadas por la Junta de Gobierno del CSIC se indica que en principio parece recomendable organizar el ICCET en dos áreas: «una científica, de investigación en construcción, y otra técnica, de desarrollo y control de calidad de la construcción; la primera de ellas podría convertirse en el embrión de un futuro centro de materiales compuestos mientras la segunda podría alcanzar un adecuado desarrollo a través de acuerdos con otras instituciones». Por eso cabe prever que vaya tomando más y más cuerpo en este instituto el estudio de materiales compuestos con matriz de cemento. De hecho últimamente se está acabando y se piensa continuar un proyecto nacional sobre materiales compuestos en la construcción; y se ha logrado un proyecto de la UE para construir un puente piloto, todo en materiales compuestos de base orgánica.

\section{Ciencia y Tecnología de polímeros}

Con más de medio siglo de vida fecunda, el ICTP, está constituido ahora por 6 departamentos de investigación: Física e ingeniería de polímeros; Fotoquímica de polímeros; Química física de polímeros; Quimica macromoecular; Química y propiedades de materiales polimeros; Química y tecnología de elastómeros; y por un grupo de Materiales compuestos y electroactivos.

Esas unidades trabajaban durante 1997 en 24 proyectos, 8 de la UE, 14 de planes nacionales, y 1 de la CAM. En el mismo año tenían 7 acciones integradas con la República de Cuba, Chile, Israel, Portugal (2 acciones) y Reino Unido (2 acciones). Y publicaban 119 artículos en revistas de índice de impacto del orden de 1,00, aunque alcanzaron el 3,50 en buen número de casos y, en uno, el 6,14 de Physical Review Letters.

Durante el mismo periodo desarrollaron también 8 contratos con firmas industriales, acabaron 7 tesis doctorales, obtuvieron dos patentes, e impartieron la $38^{\mathrm{a}}$ edición del Master en Ciencia y Tecnología de 


\section{Ciencia y Tecnología de materiales}

Polímeros en colaboración con la Confederación Española de Empresarios de Plásticos y el Consorcio Nacional de Industrias del Caucho. Ese mismo año el ICTP expidió 25 certificados de aptitud para optar a los diplomas de Especialización y Alta Especialización en Plásticos y Caucho, que otorga el Ministerio de Educación y Cultura; continuaba editando la revista mensual Plásticos modernos, que celebra ahora sus bodas de oro; y preparaba el Anuario de Plásticos, que no se editaba desde 1990.

Las líneas de investigación que cultiva el ICCTP son muchas y cubren tanto las cuestiones más básicas como las de interés tecnológico. Entre otras, cabe mencionar la preparación de nuevos materiales con propiedades físicas de gran interés (resistencia térmica, cristales líquidos); los estudios de cinetoquímica y de propiedades de los nuevos materiales sólidos y en disolución, especialmente las propiedades estructurales y conformacionales; el estudio también de las relaciones de la microestructura con las propiedades; y el diseño y desarrollo tanto de materiales compuestos como el de biopolímeros.

Entre los proyectos en curso durante 1998 que han logrado mejor aceptación de las entidades financiadoras, están los reseñados a continuación. Los tres primeros, de la UE; el último, financiado por una fundación privada; y los demás, de planes nacionales:

1) Preparación de nuevas poliolefinas con propiedades termoplásticas interesantes, por polimerización catalítica de olefinas cíclicas, tanto mediante reacciones de adición como de metátesis con apertura de anillo; y caracterización de los materiales obtenidos para determinar su estabilidad, rigidez, plasticidad, resistencia a la tracción, tenacidad y durabilidad.

2) Método para seguir, in situ y de modo no invasivo, la curación de diversos adhesivos (tipo epoxi, fenólicos, acrílicos, poliuretanos) por medio de la detección de la emisión de fluorescencia de sondas muy intensas, que permiten determinar tensiones térmicas residuales, grado de envejecimiento, corrosión, absorción de agua, fotodegradación y, en sistemas multicomponentes, extensión del daño interfacial.

3) Determinación de correlaciones entre estructura y propiedades electrónicas. Se ha logrado establecer el papel decisivo de las configuraciones locales terminales de secuencias tácticas y de las conformaciones posibles en aquéllas, como puntos de localización de cargas de espacio. Los resultados obtenidos pueden aplicarse a otros polímeros, principalmente derivados de polipropileno y de cloruro de polivinilo, y acoplar las técnicas de medida de cargas de espacio con las de medida de transiciones térmicas. 
4) Estudio de los aspectos estructurales y conformacionales que controlan la naturaleza de las mesofases de nuevos cristales líquidos, su estabilidad y los mecanismos de su formación en función de la historia térmica, así como su capacidad para adoptar orden tridimensional en condiciones determinadas y controladas. Se estudia la posibilidad de modificación microestructural de propiedades de materiales polímeros termoplásticos sólidos, mediante mezcla y reacción con poliésteres termotrópicos sintetizados y comerciales. Con ese objeto se ha analizado la interacción, la adhesión y la separación de fases, en función de la estructura de los componentes, la composición, y el tratamiento térmico.

5) Obtención y caracterización de nuevos polímeros que puedan sustituir, a ser posible con ventajas, a materiales utilizados en tecnologías avanzadas, especialmente como materiales barrera (membranas para separación de gases, ultrafiltración, ósmosis inversa); o como biomateriales (materiales compuestos, hidrogeles, productos para dosificación retardada y prótesis para implantaciones quirúrgicas).

6) Preparación de materiales compuestos polímeros. Se han sintetizado resinas de cianoéster con estructura de poliétercetona, para el tratamiento superficial de las fibras que se incorporarán como refuerzos en esos materiales. Las resinas tienen simetría, longitud y temperatura de curado muy diversas, de modo que cabe elegir la más apropiada para la temperatura del material a modificar. De otra parte pueden usarse como materiales de resistencia térmica elevada, pues tienen la misma resistencia termooxidativa que las polietercetonas aromáticas lineales del comercio, con la ventaja de que se pueden procesar a temperaturas inferiores y conservar sus propiedades por encima de la transición vítrea. A la vez se han estudiado varios caolines naturales y calcinados, en orden a abordar futuras modificaciones con silanos. Se ha continuado estudiando la adición de fibras de carbono y de poliéster oxidadas a matrices elastoméricas funcionalizadas, caucho natural epoxidado y caucho nitrílico carboxilado; y se ha determinado el efecto sobre la vulcanización de estos elastómeros con distintos sistemas de entrecruzamiento.

7) Síntesis y estudios cinéticos de la polimerización radical de nuevos monómeros acrílicos y metacrílicos con grupos funcionales hidrofílicos en cadenas laterales de gran longitud o volumen. Se han estudiado las propiedades estructurales, mecánicas y dieléctricas de todos los polímeros preparados, especialmente los que contienen anillos de 1,3dioxano. También se ha estudiado a fondo la difusión de diversos gases (oxígeno, nitrógeno, dióxido de carbono, argon) en membranas preparadas con varios de estos polímeros. 
8) Se apunta a tres objetivos: obtener polímeros superabsorbentes; preparar sistemas macromoleculares con arquitectura controlada; y tratar de degradar poliolefinas de modo termooxidante. Hasta ahora se han preparado homo- y co-polímeros de ácido acrílico con diferentes grados de neutralización, poliestireno y copolímeros de estireno con monómeros acrílicos utilizando la polimerización radical mediante transferencia atómica, $\mathrm{y}$, mediante fraccionamiento de polipropileno, muestras de isotacticidad comprendida entre el 20 y el 96 por ciento.

9) Preparación por dos métodos de polímeros entrecruzados a base de policloruro de vinilo. Primero, mediante reacción en estado fundido con organosilanos, en un solo paso que, tras optimizar el proceso, ha conducido a materiales con buenas propiedades mecánicas y térmicas. En el segundo método, de dos pasos, se modifica primero el polímero mediante el injerto de un reactivo nucleófilo portador de grupos $\mathrm{OH}$; y luego se entrecruzan las cadenas por reacción de los $\mathrm{OH}$ con diisocianato. La caracterización de los polímeros modificados y de las redes derivadas ha permitido establecer relaciones entre la estructura de los polímeros y sus propiedades, que han resultado ampliamente mejoradas respecto al polímero de partida. El estudio de las propiedades de transporte de fluidos en los nuevos polímeros muestra que cabe modificarlas en función del grado de entrecruzamiento y el número de grupos $\mathrm{OH}$ presentes en las redes.

10) Desarrollo de baterías recargables de litio mediante la preparación de nuevos electrodos y electrolitos: cátodos en los que el material activo es un compuesto de inserción de vanadio y manganeso; ánodos basados en diferentes tipos de coque; y electrolitos polímeros de conductividad iónica elevada, haciendo compatibles física y termodinámicamente poliéteres de conocida eficacia conductora.

11) Búsqueda de formulaciones acrílicas que presenten mejores propiedades mecánicas (tenacidad, resistencia a la fatiga), más capacidad de integración ósea y mejor biocompatiblidad que las utilizadas en la práctica quirúrgica actualmente. Se ha estudiado la tenacidad a fractura y la resistencia a fatiga de cementos polimerizados con activadores de baja toxicidad; la adición de nuevos sistemas radioopacos basados en compuestos aromáticos yodados, y su anclaje a sistemas polímeros; y la sustitución parcial de la fase sólida por componentes cerámicos reabsorbibles basados en fosfatos de calcio y en biovidrios. Este proyecto se realiza en colaboración con la Facultad de Química de San Sebastián y la Escuela de Ingenieros Industriales de Barcelona.

12) Síntesis y caracterización de polipropilenos modificados químicamente. Se ha prestado especial atención al injerto de ácido p-fe- 
nilén-bismaleámico y al estudio de los procesos de síntesis en disolución. Se estudia el papel que desempeñan estos productos como agentes modificadores de propiedades en materiales compuestos basados en polipropileno, y mezclas con talco, mica, poliamidas y fibras cortas de carbono, de las cuales se ha iniciado la caracterización superficial.

13) Estudio de fenómenos de relajación estructural en diversos polímeros de interés tecnológico y de la repercusión del envejecimiento físico en las propiedades (térmicas, mecánicas, viscoelásticas) que condicionan sus aplicaciones prácticas.

14) Preparación y caracterización de cristales líquidos polímeros de cadena tanto principal como lateral, que presenten mesofases $\mathrm{S}_{\mathrm{c}}$ * $\mathrm{u}$ otras equivalentes de tipo inclinado; y estudio de sus propiedades ferroeléctricas, ópticas y mecánicas, así como de las relajaciones viscoelásticas $\mathrm{y}$ dieléctricas.

Tanto el interés de estos proyectos como los demás datos sobre el ICTP mencionados, son signos de madurez institucional que se apoya en una larga tradición de buen hacer. El ICTP ha sabido combinar el trabajo de investigación básica con el de carácter más aplicado, las contribuciones de valor más fundamental con la transferencia de resultados a la industria.

Las vinculaciones del ICTP con el sector productivo no se limitan a las empresas que nacieron gracias a resultados obtenidos en el Instituto, porque éste presta habitualmente su asistencia científico-técnica a buen número de firmas industriales. Además de los contratos mencionados antes, en la memoria de 1997 se consignan hasta 212 informes y dictámenes de carácter científico-técnico emitidos a petición de industrias. Esos informes reflejan los resultados obtenidos tras análisis, determinaciones y ensayos de más de sesenta tipos diferentes.

\section{Cerámica y Vidrio}

El ICV, con sede actual en Arganda del Rey, km. 24,3 de la antigua carretera de Valencia, está proyectando su traslado a un edificio en construcción que tendrá una superficie de $4.500 \mathrm{~m}^{2}$ y se asienta en una parcela de $6.000 \mathrm{~m}^{2}$ del Campus Universitario de Cantoblanco, en el mismo solar que alberga ahora los Institutos de Biotecnología, de Catálisis y Petroleoquímica, y de Ciencia de Materiales de Madrid.

A lo largo de 1998 se han acabado en el ICV cinco tesis doctorales. $\mathrm{Y}$ los artículos publicados durante 1997 en revistas científicas fueron 61: 12, en el Boletín de la Sociedad Española de Cerámica y Vidrio; 


\section{Ciencia y Tecnología de materiales}

49 en revistas de índice de impacto por término medio 1.0; uno más en Journal of Analytical Atomic Spectrometry; y otros dos en Chemistry of Materials, revistas éstas de índice de impacto más elevado, 3,60 y 3,27 respectivamente.

Ha crecido respecto a años anteriores el número de proyectos de investigación en curso, con lo que esto viene suponiendo de transferencia de tecnología a las empresas y de aumento de ingresos del Instituto. El ICV se relaciona con el sector industrial a través de la Sociedad Española de Cerámica y Vidrio. Durante 1997 se contrataron 20 trabajos con 19 industrias. Entre los proyectos que se mencionan a continuación, los tres primeros son proyectos de la Unión Europea, el último, de la CAM, y los demás, del Plan Nacional de Materiales.

1) Búsqueda de vías innovadoras para obtener de modo reproducible y económicamente rentable, utilizando materias primas naturales de bajo coste, materiales refractarios de alto contenido en alúmina con espinela y hexaaluminato de calcio como segundas fases, para aplicaciones estructurales.

2) Colaboración para poner en marcha una pila de combustible de carbonatos fundidos, de $100 \mathrm{~kW}$. El ICV tiene confiado el desarrollo de un sello estanco, para el distribuidor de gases, que produzca uniones estables, resistentes al ataque por carbonatos fundidos y que impida la migración del electrolito entre celdas.

3) Preparación y análisis, en colaboración con 15 laboratorios de la UE, de un vidrio patrón con contenidos certificados de flúor, cloro, cromo, cobalto, arsénico, selenio, cadmio, antimonio, bario y plomo.

4) Obtención de recubrimientos vítreos de unos $10 \mu \mathrm{m}$ de espesor, obtenidos mediante vía sol-gel, inmersión y deposición electroforética, con los que se evita la corrosión de aceros inoxidables y esmaltes frente a agentes ácidos o básicos.

5) Síntesis de nuevos materiales cerámica-metal, que se utilizarán en la preparación de electrodos para pilas poliméricas de combustible (metano), con vistas a su aplicación al futuro coche eléctrico.

6) Estudio de diagramas de equilibrio de los sistemas $\mathrm{Al}_{2} \mathrm{O}_{3}-\mathrm{MgO}$ $\mathrm{CaO}, \mathrm{MgO}-\mathrm{SiO}_{2}-\mathrm{CaO}-\mathrm{ZrO}_{2}, \mathrm{ZrO}_{2}-\mathrm{MgO}-\mathrm{CaO}$, para aplicarlo al diseño de materiales estructurales de temperatura elevada. Se ha logrado preparar mullita de alta pureza y se ha solicitado una patente de invención. También se han logrado materiales con gradiente constitucional que podrían tener aplicaciones como abrasivos o como soportes para catalizadores. $\mathrm{Y}$ se han obtenido fibras de $\mathrm{Al}_{2} \mathrm{O}_{3}$ y de $\mathrm{Si}_{3} \mathrm{~N}_{4}$.

7) Preparación de nuevos vidrios basados en óxidos de metales pesados que unan, a su comportamiento óptico no lineal y transmisión 


\section{Isidoro Rasines}

alta en IR, buenas prestaciones mecánicas, térmicas y químicas, para poder aplicarlos en dispositivos fotónicos. Se estudian los sistemas $\mathrm{Nb}_{2} \mathrm{O}_{5}-\mathrm{Bi}_{2} \mathrm{O}_{3}-\mathrm{PbO}, \mathrm{Nb}_{2} \mathrm{O}_{5}-\mathrm{GeO}_{2}-\mathrm{PbO}$ y $\mathrm{Nb}_{2} \mathrm{O}_{5}-\mathrm{Ga}_{2} \mathrm{O}_{3}-\mathrm{PbO}$.

8) Diseño de un eutéctico del sistema wolastonita-fosfato tricálcico. Se ha estudiado su bioactividad en suero fisiológico artificial. Así se ha desarrollado el primer miembro de una nueva familia para la que se ha registrado la marca internacional Bioeutécticos, un material bioactivo que permite, al colonizarse, su osteointegración.

9) Preparacíón y evaluación de materiales cerámicos basados en $\mathrm{Li}_{\mathrm{x}} \mathrm{Ni}_{1-\mathrm{x}} \mathrm{O}$ hexagonal y en las perosquitas $\mathrm{Sr}(\mathrm{Fe}, \mathrm{Ti}) \mathrm{O}_{3}$ y $\mathrm{LaNiO}_{3}$, para su utilización como electrodos bi- o mono- funcionales en pilas de combustible de alta temperatura. Una vez obtenidos y caracterizados polvos nanométricos muy homogéneos y reactivos, se han sinterizado para estudiar su conductividad eléctrica. Se proyecta determinar las propiedades reológicas de suspensiones preparadas con estos polvos, con vistas a su empleo como electrodos en celdas reales de combustible.

10) Preparación de buzas de circona porosa y microestructura formada por una dispersión uniforme de circona tetragonal en una matriz de circona cúbica, que confiere al producto resistencia al choque térmico y a la abrasión por metal fundido. Después de elegir las materias primas, los métodos de homogeneización y compactación, los aditivos de prensado, y el ciclo térmico adecuados, se han logrado preparar en el laboratorio. Actualmente la empresa colaboradora está fabricando esas buzas, que se han ensayado con éxito en varias acerías.

11) Estudio del efecto dimensional en las aplicaciones y propiedades de materiales ferroeléctricos con pérdidas dieléctricas bajas. Se han aplicado métodos de control de crecimiento de grano mediante adsorción superficial de dopante por las partículas cerámicas ferroeléctricas. Se han obtenido y estudiado muestras de polvos submicrónicos de óxidos sin porosidad intrapartículas, idóneos para los procesos de modificación superficial.

12) Ensayo de técnicas diversas para conformado de piezas y recubrimientos de materiales cerámicos: la de deposición electroforética, para obtener sustratos autosoportados y/o recubrimientos, por ejemplo recubrimientos de óxidos cerámicos sobre electrodos de níquel; el moldeo por inyección a baja presión, que ha permitido poner a punto un método de bajo coste para el conformado de piezas de forma compleja; y el colaje en cinta, con nitruro de silicio y óxidos cerámicos como materiales de estudio.

13) Búsqueda de electrolitos y electrodos para baterías recargables. Se ha logrado obtener vidrios de óxidos de vanadio y teluro como cátodos para baterías con ánodo de litio o coque y un electrolito basado en carbonato de propileno con perclorato de litio. Estas baterías logran 


\section{Ciencia y Tecnología de materiales}

una excelente ciclabilidad, pues retienen el 90 por ciento de la capacidad después de 10 ciclos. Los vidrios mencionados pueden, por tanto, aplicarse en el diseño de baterías recargables.

14) Preparación de materiales cerámicos abrasivos y con alta resistencia al choque térmico. Se están desarrollando materiales estructurales con matriz alúmina o mullita y partículas de segundas fases como hexaaluminato de calcio o titanato de aluminio, con tamaños controlados, desde las nano- a las micro- partículas. También se desarrollan materiales multicapa tolerantes al daño.

15) Estudio de las uniones cerámica-cerámica y cerámica-metal en materiales de nitruro de silicio con varios aditivos. Las uniones se han hecho por interposición bien de metales o aleaciones, bien de capas de óxidos, y mediante vidrios de oxinitruro de silicio. Se ha desarrollado un equipo nuevo para caracterizar estas uniones.

16) Obtención y caracterización de materiales nanocompuestos híbridos con diferentes proporciones de material orgánico e inorgánico, para establecer relaciones entre microestructura y propiedades.

17) Preparación de membranas cerámicas para separación de gases, con el propósito de contribuir a mejorar el medio ambiente donde se usen el gas natural o los productos de gasificación del carbón como fuentes de energía. Se están desarrollando soportes porosos con diámetros del orden de los nanometros y se están haciendo ensayos de deposición en fase de vapor sobre soportes no porosos.

El ICV ha colaborado con grupos de otros países en varias acciones coordinadas o asimilables: Argentina, Francia (tres acciones), Italia y Portugal. Y ha logrado que se aprueben y financien por la Agencia Española de Cooperación Internacional dos Redes Iberoamericanas: una, sobre Ciencia y Tecnología de Materiales Vitreos, con 12 centros de 7 países (Argentina, Brasil, Colombia, España, México, Portugal y Venezuela); la otra, sobre Materiales electrocerámicos, con 22 grupos de 10 países: Argentina, Brasil, Colombia, Costa Rica, República de Cuba, Ecuador, España, México, Portugal y Venezuela. La primera inauguró sus reuniones del 13 al 15 de octubre de 1997 en Monterrey, México, donde tuvo un Seminario-Taller sobre situación de la industria y la investigación vidriera en Iberoamérica. La segunda ha facilitado la movilidad entre investigadores, de modo que actualmente hay tres doctorandos en el ICV financiados por la Agencia Española de Cooperación Internacional.

En este momento se han consolidado en el ICV líneas que deberán mantenerse y completarse mejorando en lo posible su calidad y capacidad de incidencia tecnológica, como un objetivo para el futuro próximo: 
cerámicas estructurales (óxidos y materiales sin oxígeno); vidrios para cátodos en baterías de litio recargables; vidrios para soldadura y unión de diversos materiales (cerámicos, vítreos, metálicos); y estudios sobre las interfases que aparecen en estas uniones. A la vez, se han iniciado líneas dirigidas a las principales necesidades del sector industrial, de las que cabe esperar mucho para un futuro próximo: materiales compuestos de matríz orgánica y vítrea reforzada con fibras y/o partículas; diseño de transductores ultrasónicos inteligentes; vitrificaicón de residuos industriales; $y$, especialmente, biomateriales.

\section{Investigaciones Metalúrgicas}

Desde su creación en 1963 el CENIM ha ido evolucionando ${ }^{1}$ al compás de los cambios operados en la industria metalúrgica: las modificaciones de los procesos clásicos de obtención de los metales y aleaciones, la extensión de la metalurgia extractiva al aprovechamiento de residuos y al reciclado de materiales, junto con la demanda de nuevos materiales para altas prestaciones, que van desde las aleaciones refractarias o superligeras, a los materiales compuestos y a los biomateriales. De los 7 departamentos con que contaba, ha pasado a agruparse ahora en 4 . A continuación se indican las líneas de investigación en las que trabaja cada uno.

Corrosión y protección: recubrimientos protectores, corrosión atmosférica, modificación superficial, biomateriales, corrosión de estructuras de hormigón armado, y físicoquímica de superficies.

Metalurgia física: procesamiento de materiales, caracterización microestructural, caracterización mecánica, pulvimetalurgia, transformaciones en estado sólido, deformación a alta temperaura, simulación de procesos de conformación, y materiales compuestos.

Metalurgia primaria y reciclado de materiales: caracterización de minerales y residuos, tratamientos mineralúrgicos, procesos metalúrgicos básicos, ingeniería y tecnología del medio ambiente industrial, reciclado de materiales y residuos, procesos de combustión, y obtención de materiales de alto valor añadido.

Ingeniería de materiales. Degradación y durabilidad: modelado y simulación de procesos, metalurgia de la soldadura y corte, mecánica de fractura de materiales soldables, tecnologías informáticas aplicadas, y tecnologías avanzadas de unión.

Además de las cuatro unidades de investigación, el CENIM cuenta con otras dos de servicio: Biblioteca y Documentación, y Mantenimiento; y, dependiendo de la Vicedirección de Servicios Técnicos, 7 unidades 


\section{Ciencia y Tecnología de materiales}

de apoyo y servicios: Análisis químicos, Delineación y Proyectos, Ensayos mecánicos, Laboratorio de Electrónica, Metalografía y Fotografía, Programación e Informática, y Taller mecánico.

Va aumentando, respecto de años anteriores, el nivel de las publicaciones. En la memoria de 1997 se consignaban 80 artículos publicados; la mayoría, en revistas de índice del orden de 1.0; y dos, en Physical Review B, de índice 2,88.

El CENIM, que tomó entre los Centros del Consejo la iniciativa de acudir a las convocatorias de proyectos de la UE, ha continuado orientando preferentemente su trabajo investigador en esa dirección y por eso es, entre los institutos del área, el que desarrolla más proyectos europeos de importancia. En la memoria de 1997 se reseñan 63; 46 seguían vigentes en 1998. De éstos 24 tenían financiación de la UE, 19 de planes nacionales, y 2 de la CAM. Hubo también una Acción con Chile. A continuación se incluyen resúmenes de los objetivos que se proponen algunos de esos proyectos.

1) Se buscan sistemas biológicos que permitan la degradación de los componentes orgánicos asociados a determinadas piezas de acero en el proceso de su fabricación.

2) Se estudia cómo obtener piezas galvanizadas recuperando tanto el calor sobrante en el proceso como los componentes químicos de interés.

3) Se determinan las propiedades reológicas de pulpas de aceite con partículas de hierro, con objeto de establecer las relaciones entre el sólido y el aceite más favorables para el transporte, manejo e inyección de estas pulpas en los hornos altos.

4) Se intenta la purificación y el tratamiento de mezclas de compuestos metálicos para obtener productos con posibles aplicaciones en la industria del acero.

5) Se ha construido en una acería de Gijón una cámara de 13 $\mathrm{m}^{3}$ de gasificación de coque y se ha perfeccionado el modelo frío preliminar emplazado en el CENIM.

6) Se determinan en 4 aceros de referencia trazas y ultratrazas, entre 0,05 y $0,5 \mu \mathrm{g} \mathrm{g}{ }^{-1}$, de 21 elementos químicos elegidos por su interés atendiendo al impacto medioambiental, sus efectos en las propiedades metalúrgicas y la necesidad de establecer patrones con elementos que no son frecuentes en los aceros.

7) Se estudia la aplicación de las redes neuronales y la lógica difusa a la modelización y control de dos hornos altos de Gijón, de modo que cabe predecir los porcentajes de óxidos de carbono, hidrógeno $\mathrm{y}$ silicio. 
8) Se han determinado las variables más influyentes en la calidad del sinter producido en una acería.

9) Se desarrollan aleaciones de aluminio, una de la familia Al$\mathrm{Fe}-\mathrm{V}-\mathrm{Si}$, utilizables en la industria aeroespacial.

10) Se trata de preparar materiales compuestos de matriz de aluminio, reforzados con partículas de polvo de intermetálicos procesados por vía pulvimetalúrgica, con aplicaciones en la industria del transporte.

11) Se propone la caracterización microestructural y mecánica de aleaciones de base hierro y níquel para la fabricación de tuberías sin soldaduras.

12) Se estudia la deformación en caliente de tres aceros con diferente contenido en titanio, 0,1 por ciento de carbono y 80 partes por millón de nitrógeno, con el fin de determinar la evolución microestructural en la laminación posterior a la colada próxima a la forma final, a cualesquiera condiciones de temperatura, deformación y velocidad de deformación que se apliquen.

13) Se ha estudiado en tres atmósferas naturales, frente a tres tipos de ensayos acelerados de corrosión y con sensores electroquímicos de diseño especial, el comportamiento de las uniones solapadas (remaches y puntos de soldadura) de acero recubierto con diferentes revestimientos metálicos.

14) Se estudiará la modificación de los recubrimientos galvanizados obtenidos sobre acero para armaduras, por inmersión en baños calientes con diferentes adiciones de níquel, con el fin de determinar la influencia de esta adición en la reactividad del acero.

Se consignan en la misma memoria 37 contratos con empresas. Tienen duración variable, de unos meses a dos años, y cuantías que van desde 300 a 6.400 miles de ptas. En la mayor parte de los casos no se limitan a la realización rutinaria de análisis o ensayos, sino que se centran sobre la resolución de problemas industriales de interés para las firmas que los suscriben.

Para un futuro próximo parece oportuno ${ }^{4}$ mantener y mejorar aún más las líneas de trabajo bien asentadas en el CENIM: producción de metales como aluminio, hierro níquel y cobre, a partir de polvos solidificados rápidamente; estudio de propiedades mecánicas de materiales metálicos (metales, intermetálicos, aleaciones) con vistas, por ejemplo, a controlar la microestructura, de modo que puedan soportar grandes tensiones; trabajos sobre corrosión; y sobre aprovechamiento $y_{0}$ freciclado de residuos. Con ese objeto puede también ser oportuno incorporar a científicos que sean capaces de iniciar a alto nivel alguna línea de investigación nueva. 


\section{Ciencia y Tecnología de materiales}

\section{Ciencia de Materiales en Aragón}

Por su condición de centro mixto, el ICMA incluye personas tanto de la UZ como del CSIC, repartidas en dos sedes, la Facultad de Ciencias y el Centro Politécnico, ambas de la UZ. Comprende el Instituto dos áreas temáticas, la de Ciencia y Tecnología de Materiales, y la de Ciencia y Tecnología Químicas, con 8 departamentos: Química de los compuestos organometálicos; Química de la coordinación y catálisis homogénea; Química orgánica; Espectroscopía de sólidos; Física del estado sólido a bajas temperaturas; Magnetismo de sólidos; Propiedades físicas de materiales con interés tecnológico; y Nuevos materiales orgánicos.

El final de la primera fase del proyecto de ampliación y remodelación de la Facultad de Ciencias ha permitido el traslado de 4 departamentos a las nuevas dependencias, resolviendo así en buena parte la falta de espacio físico, que constituía uno de los problemas más acuciantes del Instituto. A comienzos del año 2.000 se espera la solución del mismo problema para otros 3 departamentos.

Para el desarrollo de sus actividades el ICMA contrata investigación que gestiona, a elección del investigador responsable, bien la UZ bien el CSIC. El anexo 2 incluye la cuantía, 216 millones, de los proyectos de investigación contratados en 1998. A continuación figuran los títulos de estos proyectos: los 6 primeros contaron con la financiación de la $\mathrm{UE}$; los 31 siguientes, de planes nacionales; y del $\mathrm{n}^{\circ} 38$ al 42, de la Diputación General de Aragón. Además, el ICMA desarrolló en el mismo año 10 convenios o contratos con industrias diversas.

1. Aplicaciones de potencia de la superconductividad.

2. Magnetismo molecular de materiales para dispositivos.

3. Aplicaciones de imanes.

4. Licuefacción de carbones con catalizadores solubles asistidos por microondas o ultrasonidos.

5. Preformas textiles multiaxiales para partes compuestas estructuralmente complejas.

6. Nuevos procesos fotoselectivos para pantallas fluorescentes planas.

7. Síntesis, estructura y reactividad de nuevos tipos de complejos polinucleares con enlaces metal-metal y ligandos polihalofenilo.

8. Síntesis, reactividad y actividad catalítica de organocomplejos de metales del grupo del platino.

9. Compuestos organometálicos polinucleares de paladio y platino. Especies homo- y hetero-metálicas. Síntesis, estructura y reactividad.

10. Tiolato complejos de oro. Síntesis dirigida y estudio de sus propiedades. 
11. Nuevos complejos de metales del grupo del platino con interacciones o enlaces metal-metal inter- o intra-moleculares.

12. Complejos de rodio(II). Caracterización estructural y reactividad.

13. Estructura y propiedades físicas de complejos con ligandos polifuncionales y de sus disoluciones sólidas moleculares.

14. Aplicaciones de la dinámica no lineal y la física computacional a sistemas modelo de interés en física de la materia condensada.

15. Diseño de sistemas metálicos homogéneos capaces de llevar a cabo la síntesis de moléculas orgánicas funcionalizadas a partir de hidrocarburos básicos.

16. Catalizadores organometálicos enantioselectivos.

17. Síntesis, estructura y propiedades de complejos mono- y polinucleares de oro o plata. Interacciones metal-metal.

18. Síntesis asimétrica de compuestos de alto interés biológico. Preparación de nucleósidos, isonucleósidos y análogos.

19. Estudio de las interacciones entre ciclos aromáticos y de carácter donor de centros aniónicos: síntesis, estructura y reactividad de nuevos complejos con ligandos perhalo-fenilo.

20. Nuevos cromóforos y sistemas redox derivados de diadas tetratiofulvalenos.

21. Texturado de superconductores cerámicos de alta temperatura por el método de zona flotante.

22. Estudio del estado mixto en superconductores de alta temperatura.

23. Catalizadores orgánicos enantioselectivos.

24. Nuevos materiales magnéticos: intermetálicos, óxidos y moleculares.

25. Síntesis, caracterización estructural, magnética y eléctrica de óxidos cerámicos conductores y magnetorresistivos.

26. Materiales masivos con magnetorresistencia y magnetoestricción gigantes.

27. Diseño molecular de nuevos catalizadores soportados: aplicación a reacciones selectivas de oxidación y cicloadición.

28. Tratamiento y puesta en forma de materiales electrocerámicos y aislantes mediante métodos electroquímicos y de fusión zonal.

29. Materiales polímeros metalomesógenos. Estudio de sus propiedades ópticas no lineales.

30. Síntesis y crecimiento de materiales eutécticos con estructuras alineadas mediante fusión zonal por láser.

31. Síntesis, estructura, estudios magnéticos y magnetoópticos de materiales moleculares y sistemas magnéticos diluidos. 
32. Diseño, síntesis y caracterización de materiales basados en organizaciones supramoleculares para aplicaciones ópticas.

33. Materiales intermetálicos con aplicaciones en imanes duros y óxidos magnéticos con propiedades magnetorresisitivas.

34. Preparación, caracterización y estudio magnético de láminas delgadas y multicapas de materiales intermetálicos y de óxidos con magnetorresistencia colosal.

35. Materiales compuestos para su uso en refuerzo o rehabilitación de edificios y de obra civil.

36. Diseño y construcción de comparadores criogénicos de corriente óptimos y su detector SQUID para aplicaciones en metrología cuántica.

37. Caracterización mediante resonancia electrónica de protón y espectroscopía Raman de materiales cerámicos conductores catiónicos con aplicación en baterías recargables y otros dispositivos.

38. Estudio y caracterización física de semiconductores magnéticamente diluidos del tipo II-VI, IV-VI y $\mathrm{II}_{-} \mathrm{III}_{2}-\mathrm{VI}_{6}$.

39. Predicción de la microestructura de las fundiciones de hierro. Influencia de los elementos de aleación y de las impurezas.

40. Fluoruros dopados con tierras raras. Tecnología, espectroscopía, realización de guías ópticas activas.

41. Espectroscopía de iones $4 f$ en vidrios fluoroclorados, bromados o yodados.

42. Aplicación de biomateriales con memoria de forma en prótesis, elementos y dispositivos en el área médica.

Entre las relaciones internacionales del ICMA destaca el trabajo de MANES, un LEA para el estudio de materiales por difracción de neutrones y radiación de sincrotrón, constituido por convenio entre el Laboratorio de Cristalografía del CNRS de Grenoble y el Departamento de Física del Estado Sólido a bajas temperaturas del ICMA En MANES se estudian propiedades de materiales magnéticos y de conductores; se aplican técnicas de difracción, de difusión y espectroscópicas a los materiales que se preparan y se estudian después en el ILL y en la IERS. A esta colaboración habitual hay que añadir la de 8 acciones integradas vigentes durante 1998.

La excelente calidad del trabajo realizado por el personal investigador del ICMA queda patente en la lista de sus publicaciones, un total de 216 durante 1998: más de la mitad, 113 artículos, en revistas con índice de impacto superior a $2.0 ;$ y 10 de los cuales, con índices muy elevados, superiores a 5.0. Por otra parte los investigadores del ICMA han participado en 11 congresos de ámbito nacional con 45 


\section{Isidoro Rasines}

contribuciones y en 65 de carácter internacional con 122; y durante 1998 se han acabado en el Instituto 11 tesis doctorales.

\section{Ciencia de Materiales en Barcelona}

En la última memoria bienal, 1996-1997, del ICMB se encuentran los hitos de la historia del Instituto desde su fundación en 1987 por el CSIC. La sede actual, en el campus de la Universidad Autónoma de Barcelona, se edificó inicialmente con un piso menos de los previstos en el proyecto. En el año 1997 se ha construido ese piso, de unos $1.000 \mathrm{~m}^{2}$ de superficie. La ampliación no sólo ha mejorado las condiciones de trabajo, sino también la apariencia del edificio.

El ICMB comprende 4 departamentos: Materiales moleculares y supramoleculares; Cristalografía y química del estado sólido; Materiales magnéticos y superconductores; y Materiales electrónicos y crecimiento de cristales. El Instituto se propone como objetivo principal el de adaptar sus líneas de investigación al entorno industrial. Por eso ha constituido la unidad de Transferencia de tecnología.

El ICMB ha iniciado una nueva expansión al proponerse construir otro edificio cercano, de $2.600 \mathrm{~m}^{2}$, en colaboración con la firma Carburos metálicos, para albergar la asociación de interés económico denominada Laboratorio de Tecnología de Materiales y Gases, MATGAS. Este laboratorio desarrollaría, entre otros, los contratos de investigación del ICMB sobre gases, con la firma mencionada: obtención y purificación; preparación y estudio en estado ultrapuro; interacciones con superficies; y preparación de membranas, materiales porosos y catalizadores, para procesos de separación y purificación. El plan de actuación, 1995-1999, del ICMB incluye los grupos de líneas de trabajo que siguen.

Materiales moleculares y supramoleculares, membranas y catalizadores. Diseño de catalizadores homogéneos. Membranas y sensores polímeros. Diseño, síntesis y estructura de materiales orgánicos con propiedades eléctricas y magnéticas. Materiales supramoleculares con propiedades ópticas y eléctricas interesantes. Estructura electrónica e inestabilidades de sólidos de dimensionalidad baja. Densidades electrónicas precisas a partir de datos de difracción de alta resolución a temperatura baja. Nuevos métodos de resolución de estructuras cristalinas por difracción de rayos $\mathrm{X}$ ordinaria y de radiación de sincrotrón.

Procesos preparativos y morfología; nanomateriales; capas delgadas; crecimiento de cristales, y materiales porosos. Depósito de capas delgadas por DQV, ablación con láser y evaporación a alto vacío. Cristalografía 


\section{Ciencia y Tecnología de materiales}

de superficies. Cristales fotónicos, de zeolitas y de óxidos mixtos para aplicaciones medioambientales. Biomateriales y cristalización industrial. Electrodos de inserción y materiales para baterías recargables. Electroquímica aplicada a la síntesis y procesado de materiales. Aerogeles: preparación, caracterización y aplicaciones.

Materiales magnéticos, electrónicos y superconductores. Preparación, estructura y propiedades. Diseño de nuevas fases superconductoras y óxidos relacionados. Síntesis de cerámicas, capas (delgadas, gruesas) de óxidos (superconductores, magnéticos). Procesado y microestructura de materiales cerámicos (superconductores de corriente crítica elevada, magnéticos). Propiedades eléctricas de materiales superconductores. Propiedades estructurales y de magnetotransporte de óxidos magnetorresistentes y de óxidos nanoestructurados. Dispositivos (superconductores, magnéticos) para aplicaciones eléctricas y aplicaciones electrónicas. Relación entre microestructura y propiedades ópticas y electrónicas de cerámicas, monocristales, y capas superconductoras. Estudio de las propiedades ópticas de heteroestructuras de semiconductores, con capas de carburo de silicio y circona.

La actividad investigadora del ICMB se ha centrado durante 1997 en 44 proyectos, cuyos títulos se indican a continuación. Los 6 primeros fueron financiados por la UE; los 18 siguientes, por planes nacionales; del $\mathrm{n}^{\circ} 25$ al 31, por la Generalitat de Catalunya; el 32, por una fundación privada; y los siguientes, por industrias diversas. Además, durante el mismo año se acabaron 12 tesis doctorales y se registraron 4 patentes.

1) Nuevos conductores moleculares: fases cristalinas y capas delgadas organizadas.

2) Transferencia electrónica y energética en sistemas modelo y sus implicaciones para la electrónica molecular.

3) Desarrollo de recubrimientos cerámicos para la zona transgingival.

4) Tecnología y propiedades ópticas de superconductores de temperatura elevada.

5) Capas delgadas funcionales de óxidos, obtenidas por técnicas de deposición química a partir de vapores organometálicos.

6) Tecnologías innovadoras para mejorar la resistencia al desgaste.

7) Sólidos moleculares y polímeros de naturaleza dendrimérica o quiral, con propiedades magnéticas, optomagnéticas y/o ópticas.

8) Anclaje de flujo y corrientes críticas en superconductores anisótropos. Magnetorresistencia en materiales magnéticos nanoestructurados.

9) Procesado de superconductores con altas corrientes críticas: desarrollo de prototipos de sistemas electrotécnicos.

10) Síntesis y caracterización de superconductores de mercurio. 
11) Magnetorresistencia en óxidos. Mecanismos básicos y desarrollo de dispositivos.

12) Propiedades ópticas de superconductores de alta temperatura crítica y materiales análogos; y caracterización por espectroscopías ópticas de capas de $\mathrm{YBa}_{2} \mathrm{Cu}_{3} \mathrm{O}_{7}$.

13) Caracterización de subóxidos de silicio crecidos por deposición química a partir de vapor para la fabricación de componentes ópticos integrados.

14) Capas delgadas de materiales orgánicos moleculares superconductores y óxidos superconductores de alta temperatura.

15) Aerogeles como material de relleno para el transporte de gases.

16) Tratamiento y puesta en forma de materiales electrocerámicos $\mathrm{y}$ aislantes mediante métodos electroquímicos y de fusión zonal.

17) Materiales superconductores y magnetorresisitivos basados en óxidos, oxinitruros y nitruros complejos de metales de transición.

18) Potenciómetros sin contactos basados en materiales magnetorresistivos.

19) Procedimiento para el recubrimiento superficial de implantes metálicos con hidroxiapatito.

20) Estructuras e inestabilidades electrónicas en metales de baja dimensionalidad.

21) Organizaciones supramoleculares con propiedades magnéticas, eléctricas y ópticas: materiales orgánicos, metalorgánicos y bifuncionales.

22) Síntesis de metalocarboranos sustituidos. Estudio de su actividad como transportadores en membranas líquidas.

23) Densidades electrónicas precisas en nuevos materiales avanzados.

24) Relación entre síntesis, estructura y propiedades de transporte en perosquitas laminares de valencia mixta.

25) Ciencia y nanotecnología de superficies.

26) Materiales moleculares con propiedades eléctricas, magnéticas y ópticas.

27) Aplicaciones de materiales magnéticos y materiales superconductores.

28) Nanosistemas magnéticos y aplicaciones.

29) Estudio local de procesos de EQV.

30) Recubrimientos duros con coeficientes de rozamiento muy bajos.

31) Complejos metálicos de carboranos para catálisis asimétrica.

32) Polímeros sintéticos quirales ópticamente activos con estructuras helicoidales. Estudio del fenómeno de la teleinducción quiral y de sus posibles aplicaciones como cristales líquidos. 
33) Cristalizaciones supercríticas como alternativa a los procesos de acabado convencionales para la obtención de pigmentos y colorantes.

34) Optimización de sistemas magnetohidrodinámicos de acondicionamiento de aguas.

35) Análisis de transformadores planos.

36) Membranas cerámicas obtenidas por deposición química a partir de vapores organometálicos.

37) Aerogeles de silicio.

38) Polimorfismo y estabilidad de fármacos.

39) Reducción electroquímica del dióxido de carbono, primer paso para su fijación industrial y papel de los óxidos de valencia mixta.

40) Desarrollo y fabricación de sistemas de interés en electricidad de potencia basados en materiales superconductores cerámicos.

41) Obtención de depósitos superconductores de $\mathrm{Bi}_{2} \mathrm{Sr}_{2} \mathrm{CaCu}_{2} \mathrm{O}_{\mathrm{x}}$ e $\mathrm{YBa}_{2} \mathrm{Cu}_{3} \mathrm{O}_{7}$ por electrodepósición y electroforesis.

42) Materiales híbridos electroactivos basados en polímeros orgánicos conductores y en óxidos de metales de transición.

43) Dispositivos fotónicos con superconductores de alta temperatura.

44) Materiales híbridos poliimida-sílice para separación de gases.

Toda la actividad investigadora del ICMB ha conducido a una producción científica elevada, tanto en calidad como en cantidad, que continúa el ritmo de crecimiento gradual observado desde la fundación del Instituto: los poco más de 40 artículos publicados en 1987 se han convertido en los 115 de 1997, de los cuales uno en Angewandte Chemie, tres en The Journal of the American Chemical Society, con índices de impactos respectivos 8.56 y 5.65 , y otros 39 en revistas de índices de impacto superiores a 2.0 .

En cuanto a relaciones internacionales, están en primer término las propias del LAE de Ciencia e Ingeniería de Materiales, constituido por el Laboratoire des Matériaux et Procedés Membranaires, de Montpellier, el Institut des Matériaux et Procédés de Odeillo y Perpignan, y el ICMB. Están también los acuerdos con el National Institute for Research in Inorganic Materials, de Tsukuba, y con el Instituto de Química Física de Chernogolovka; y los contactos con diversos centros de prestigio con vistas a los programas de la UE. También, las 11 acciones integradas vigentes en 1997: Alemania, Chile, Francia (4 acciones), Italia, Japón, México, Portugal, y Polonia; y los congresos internacionales organizados por científicos del Instituto: Euroboro 97, el vigésimo cuarto simposio internacional de química macrocíclica, Euro CVD-12, la cuarta conferencia europea de superconductividad aplicada; 
la segunda reunión europea de densidad de carga, y la séptima conferencia europea de difracción de polvo.

Para el futuro próximo el ICMB se propone ampliar las líneas actuales desarrollándolas en tres direcciones: lograr que tengan más soporte teórico, como ha sugerido el Comité científico asesor; aumentar el número de técnicas preparativas y de caracterización disponibles; y conseguir que las líneas de investigación cobren más relieve industrial, mediante el desarrollo de prototipos y dispositivos con base en los materiales que se preparan y caracterizan en el Instituto.

\section{Ciencia de Materiales en Madrid}

La tabla 1, tomada en su mayor parte de la memoria de 1998, permite formarse una idea de conjunto de la labor realizada por el Instituto. En la misma memoria se relacionan, con los índices de impacto respectivos, los 248 artículos publicados en revistas incluidas en los informes del ISI, entre los cuales 94 en revistas con índice superior a 2,0. Encabezan esa lista 2 trabajos publicados en Angewandte Chemie y 15 en Physical Review Letters, con índices de impacto 8,56 y 6,14 respectivamente.

El personal del ICMM está integrado en los 9 departamentos, 9 unidades de apoyo generales y 8 unidades de apoyo instrumental que se relacionan a continuación.

Departamentos: Física e Ingeniería de superficies; Intercaras y crecimiento; Materiales ferroeléctricos; Materiales moleculares y compuestos de intercalación; Materiales particulados; Propiedades ópticas, magnéticas y de transporte; Síntesis y estructura de óxidos; Sólidos iónicos; y Teoría de la materia condensada.

TABLA 1. Resumen de la actividad científica del ICMM durante el año 1988

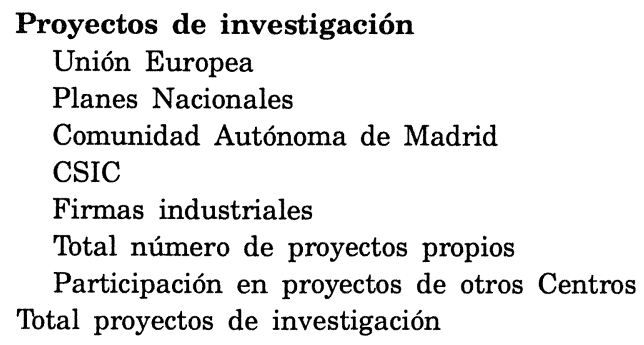




\section{Ciencia y Tecnología de materiales}

\begin{tabular}{||r|r|}
\hline Producción científica & \\
Líneas de trabajo & 75 \\
Artículos de revistas con índice de impacto definido & 248 \\
Artículos en otras revistas & 17 \\
Artículos en publicaciones colectivas & 18 \\
Libros & 2 \\
Tesis doctorales acabadas & 11 \\
Patentes en activo & 27 \\
Patentes en explotación & 13 \\
Seminarios organizados en el Instituto & 57 \\
Seminarios impartidos en otros centros & 33 \\
Comunicaciones en reuniones científicas & 145 \\
\hline Cooperación científica & \\
Unidades Asociadas & 6 \\
Convenios con entidades de otros países & 22 \\
Estancias más de 15 días de científicos del ICMM en otros países & 22 \\
Estancias en el ICMM de más de 15 días de científicos de otros países & 44 \\
\hline
\end{tabular}

Unidades de apoyo generales: Administración y secretaría; Biblioteca; Electrónica; Mantenimiento del edificio; Proyectos y delineación; Red informática; Reprografía; Taller de vidrio; y Taller mecánico.

Unidades de apoyo instrumentales: Análisis químico; Análisis térmico; Difracción de rayos X; Espectroscopía IR; Magnetómetro; Microscopía electrónica de transmisión; Monocristales (orientación, corte y pulido); y Resonancia magnética nuclear.

Es responsable el ICMM de 3 proyectos institucionales relacionados con el acceso a grandes instalaciones. El primero, con un importe total de 186 millones de ptas. para el periodo 1995-1998, consiste en la línea de luz francoespañola de tercera generación, sobre el anillo Super-Aco, del LURE. El segundo, con 121 millones durante el periodo 1998-1999, para instalar una línea española de experimentación en la IERS de Grenoble; y el tercero, de 97 millones, para estudiar la viabilidad y diseñar la línea de luz española en la misma instalación de Grenoble.

Entre los proyectos actualmente en curso están los que siguen. Los 7 primeros, financiados por la UE; los 44 siguientes, por planes nacionales; los 4 que van a continuación, por la CAM; y los 8 últimos, por compañías industriales.

1) Tintas de alta temperatura y sistemas de impresión computarizados para grabar y decorar productos acabados y medioacabados.

2) Desarrollo de materiales de mullita-circonia mediante sinterización reactiva para aplicaciones de alta temperatura.

3) Óptica de campo cercano para nanotecnología. 


\section{Isidoro Rasines}

4) Microfabricación con tecnología sol-gel asistida con ultravioleta.

5) Membranas para separación y limpieza de gases en plantas de ciclo combinado de gasificación integrada.

6) Transporte cuántico de electrones sometidos a campos dependientes del tiempo.

7) Estructura atómica y electrónica de compuestos de boro, carbono y nitrógeno.

8) Aplicación de nanotecnologías en el espacio: nanosensores para el satélite NANOSAT (fases $\mathrm{C}$ y D).

9) Láminas ferroeléctricas para detectores de IR y memorias no volátiles obtenidas a partir de soluciones de organometálicos.

10) Optimización de la magnetorresistencia gigante en perosquitas de $\mathrm{Mn}, \mathrm{Co}, \mathrm{Ru}$ y multicapas metálicas.

11) Fabricación y caracterización de sistemas nanoestructurados para su utilización como memorias de alta densidad.

12) Demostración de sistemas láser y de deflectores ópticos integrados sobre fosfuro de indio.

13) Respuesta eléctrica de materiales compuestos para electrodos positivos derivados de $\mathrm{LiMn}_{2} \mathrm{O}_{4}$ y de electrolitos tipo nasicon. Aplicación a baterías de litio en estado sólido.

14) Membranas cerámicas para la separación de gases.

15) Láminas delgadas ferroléctricas de tipo perosquita para dispositivos microelectrónicos.

16) Estudio de problemas de superficies y crecimiento.

17) Obtención, caracterización y aplicaciones tecnológicas de aleaciones estructural y magnéticamente multifásicas.

18) Materiales compuestos cerámica/metal/laminados y con función gradiente.

19) Histéresis, relajación y magnetotransporte en materiales nanoestructurados.

20) Materiales de intercalación de litio y de especies orgánicas basadas en óxidos y sulfuros de metales de transición para dispositivos electroquímicos.

21) Preparación de materiales ferropiezoeléctricos basados en nuevos óxidos con estructura tipo perosquita laminar para aplicaciones en altas temperaturas.

22) Materiales cerámicos para baterías recargables y otros dispositivos.

23) Cristales de luz: propiedades ópticas.

24) Recubrimientos duros de composición gradual. Capas depositadas por sputtering magnetron y haz de iones. 
25) Desarrollo de un sensor solar y un magnetómetro para el satélite NANOSAT empleando micronanotecnología.

26) Relaciones entre estructura atómica y propiedades electrónicas de intercaras semiconductoras.

27) Propiedades físicas de nanoestructuras.

28) Relación estructura-propiedades en materiales $d$ - $f$ (boratos, germanatos, boronitruros); y en germanatos microporosos.

29) Síntesis de recubrimientos duros de nitruros de boro y carbono mediante técnicas de DQV asistidas por plasma.

30) Conductividad iónica de materiales tipo nasicon y óxidos mixtos basado en $\mathrm{Bi}_{2} \mathrm{O}_{3}$, en relación a la estructura cristalina y composición química.

31) Estudio de la dispersión de ondas clásica y cuánticas en estructuras aleatorias.

32) Nuevos vidrios preparados mediante procesos sol-gel con aplicaciones en óptica y electroóptica.

33) Estructura atómica y electrónica en sistemas de baja dimensionalidad.

34) Heteroestructuras metálicas, crecimiento, propiedades de transporte y caracterización magnética.

35) Preparación a altas presiones y estudio de óxidos de metales de transición en estados de valencia no usuales.

36) Preparación de materiales con porosidad controlada para aplicaciones en tecnología de adsorción, separación y catálisis; desarrollo a escala preindustrial.

- 37) Estructura atómica y propiedades magnéticas en sistemas de baja dimensionalidad.

38) Preparación y propiedades de sistemas magnéticos nanoparticulados y nanocristalinos.

39) Preparaciones a altas presiones y estudio de cupratos con estructura en capas infinitas y de óxidos de metales de transición en estados de valencia inusuales.

40) Geles magnéticos inorgánicos preparados mediante métodos sol-gel.

41) Mecánica estadística de modelos simples para sistemas complejos.

42) Propiedades espectroscópicas y magnéticas en boratos y germanatos con tierras raras: relaciones con la estructura cristalina.

43) Superficies de Fermi e inestabilidades electrónicas en sistemas de baja dimensionalidad.

44) Simulación de procesos dinámicos con integrales de camino de Feynman. 
45) Mecánica estadística de sistemas complejos-95.

46) Síntesis en grandes cantidades, caracterización y aplicaciones de materiales soportados en arcillas

47) Mecánica estadística de sistemas complejos-97.

48) Obtención de pinturas absorbentes de microondas.

49) Control de potencia en la producción de óxidos nanométricos mediante pirolisis láser.

50) Química organometálica del paladio y el rutenio. Nuevas reacciones y aplicaciones en síntesis orgánica.

51) Materiales y dispositivos de electrones fuertemente correlacionados.

52) Nuevos elementos sensores y soportes de grabación magnética. Fabricación de hilos y nanohilos y caracterización de microscopía de fuerzas magnéticas.

53) Preparación de materiales ferropiezoeléctricos basados en nuevos óxidos con estructura tipo perosquita laminar para aplicaciones en altas temperaturas.

54) Optimización de láminas piezoeléctricas heteroepitaxiales para filtros resonantes de radio-frecuencia.

55) Respuesta eléctrica de materiales compuestos derivados de $\mathrm{LiMn}_{2} \mathrm{O}_{4}$. Aplicación como electrodo positivo a baterías recargables de litio.

56) Evaluación de la resistencia a la hidruración de vainas de zircaloy con recubrimientos de óxidos cerámicos protectores.

57) Apoyo tecnológico a medidor de impedancias eléctricas-III.

58) Propagación de luz en medio densos con especial énfasis en la detección de objetos ocultos.

59) Evaluación de la capacidad desodorante de sepiolitas y de otros minerales adsorbentes.

60) Preparación y caracterización de muestras de esmectitas férricas.

61) Materiales arcillosos de naturaleza esmectítica de alta densidad de carga.

62) Apoyo tecnológico a medidor de impedancias eléctricas-II.

63) Recubrimientos cerámicos sobre antorchas metálicas para su aplicación en la fundición de aluminio.

En cuanto a colaboraciones, el ICMM tuvo 22 acciones integradas con científicos de Argentina (3 acciones), Brasil, Bulgaria (2 acciones), Canadá, República de Cuba, Chile, Francia (4 acciones), Italia, Marruecos, México, Reino Unido (3 acciones), Rumania, y UE (2 acciones). $\mathrm{Y}$ tres de sus departamentos colaboraron habitualmente con los 7 grupos universitarios que tienen constituidas unidades asociadas y se indican a continuación. 


\section{Ciencia y Tecnología de materiales}

En cooperación con el Departamento de Teoría de la Materia Condensada, los grupos de: Teoría de la Materia Condensada, del Departamento de Física de Materiales de la Universidad del País Vasco; Matemáticas Aplicadas a la Materia Condensada, del Departamento de Matemáticas de la Universidad Carlos III de Madrid; Teoría de la Materia Condensada, del Departamento de Física Aplicada de la Universidad de Alicante; y Química Cuántica, del Departamento de Química Física de la misma Universidad.

En cooperación con el Departamento de Propiedades Ópticas, Magnéticas y de Transporte: el Grupo de Acústica Arquitectónica, del Departamento de Física Aplicada de la Universidad Politécnica de Valencia; y el Instituto de Magnetismo Aplicado Salvador Velayos, de la Universidad Complutense de Madrid y la RENFE.

Y en cooperación con el Departamento de Síntesis y Estructura de Óxidos, el Laboratorio de Bajas Temperaturas y Superconductividad, de la Facultad de Física de la Universidad de Santiago de Compostela.

\section{Ciencia de Materiales en Sevilla}

Una vez superados los inconvenientes del cambio de sede, desde los locales de las Facultades de Química y de Farmacia y los de los anexos del Instituto de Recursos Naturales del CSIC, a su actual emplazamiento en el edificio del Centro de Investigaciones Científicas Isla de la Cartuja, comienzan a sentirse las ventajas del traslado: en particular, se dispone de instalaciones nuevas y más completas, y ahora resulta más fácil la posibilidad de interaccionar con otros investigadores.

El ICMS comprende 4 unidades de investigación y una de apoyo. Tres de las primeras, Síntesis y reactividad de materiales, Superficies e interfaces, y Estructura y catalizadores, tienen su sede en el Centro de Investigación Científica Isla de la Cartuja, y la otra, Física de materiales, en la Facultad de Física. A lo largo de 1998 el ICMS ha trabajado en 26 proyectos. A continuación se indican los títulos. Los 6 primeros fueron financiados por la UE; y el resto, por planes nacionales.

1) Optimización del comportamiento a la oxidación a alta temperatura de los aceros inoxidables convencionales mediante la aplicación superficial de elementos de tierras raras.

2) Poliuretanos inteligentes para componentes con gradientes funcionales eléctricos y térmicos.

3) Desarrollo y aplicación de nuevos recubrimientos de nitruro de carbono en herramientas de corte. 
4) Capas y membranas inorgánicas porosas para eliminar la polución.

5) Transformación de minerales.

6) Diseño de aceros inoxidables bajos en níquel.

7) Optimización del comportamiento a la oxidación a altas temperaturas de aceros inoxidables convencionales mediante la aplicación de elementos lantánidos.

8) Desarrollo de métodos de síntesis y procesado de fibras cortas monocristalinas de mullita, whiskers, como fase reforzante en materiales compuestos de matriz cerámica con utilidad como refractarios avanzados.

9) Desarrollo de nuevos métodos de depósito de capas finas en forma de membranas con aplicaciones para la eliminación catalítica de contaminación gaseosa y como sensores de gases.

10) Comportamiento mecánico de materiales multicapas del sistema $\mathrm{Al}_{2} \mathrm{O}_{3}-\mathrm{ZrO}_{2}$.

11) Comportamiento mecánico de nanocerámicas estructurales de $\mathrm{ZrO}_{2}$ y $\mathrm{Al}_{2} \mathrm{O}_{3}$.

12) Microestructura, propiedades eléctricas y magnéticas de aleaciones tipo finemet.

13) Preparación y caracterización de nuevos aceros inoxidables austeníticos adaptados a altas prestaciones refractarias.

14) Generación de nuevas fases a partir de acuocomplejos de cationes $4 f$ intercalados en aluminosilicatos.

15) Limpieza de materiales ornamentales con medios no agresivos

16) Influencia de cambios composicionales en la microestructura y propiedades magnéticas de aleaciones nanocristalinas basadas en FeSiB.

17) Estudio teórico-experimental de estructura, dinámica y reactividad de sistemas en disolución.

18) Nueva síntesis indirecta de aluminosilicatos con estructura de mesoporos orientada por una fase de surfactante.

19) Influencia en la tensión uniaxial y la dinámica sobre las propiedades térmicas en las proximidades de transiciones de fase ferroelásticas e inconmensuradas.

20) Nuevos catalizadores soportados para descontaminación de gases de escape de vehículos con alimentación neta oxidante.

21) Métodos de procesado vía sol-gel para la preparación de óxidos mixtos como catalizadores ecológicos.

22) Control de la microestructura en materiales nanoestructurados a través de los métodos de preparación de análisis térmico a velocidad de reacción constante y mecanosíntesis. 


\section{Ciencia y Tecnología de materiales}

23) Nuevos sistemas de hidróxidos y óxidos laminares como precursores de óxidos tridimensionales.

24) Preparación mediante reacciones químicas en aerosoles y propiedades ópticas de pigmentos cerámicos de matriz sílice-alúmina.

25) Control de la microestructura en materiales nanoestructurados a través de los métodos de preparación: condensación en atmósfera de gas inerte y método electroquímico.

26) Difracción y dinámica de baja frecuencia en sólidos.

A la vez, el ICMS ha trabajado durante 1998 en 6 convenios o contratos suscritos con entidades diversas; y en 12 acciones integradas con: Alemania, República Argentina, República Checa, Francia, Hungría, Polonia (2 acciones), una acción COST en la que participaban 11 países; y la OTAN (4 acciones). También a lo largo del mismo año el ICMS ha emitido 24 informes técnicos, dos para una industria de pinturas; y el resto, sobre muestras de imágenes sagradas, para la Consejería de la Junta de Andalucía. Los proyectos relacionados, junto con los trabajos realizados que se acaban de mencionar, suponen un importe de investigación contratada, en miles de pesetas, de 129.075, procedentes de las fuentes de financiación que se indican en el anexo 2.

Durante el mismo año se acabaron en el ICMS 7 tesis doctorales y 2 de Licenciatura; los científicos del Instituto impartieron 9 conferencias y se tuvieron 12 seminarios dirigidos por científicos visitantes; se enviaron 30 comunicaciones a reuniones científicas, 27 a congresos internacionales y el resto, a reuniones nacionales. A la vez, la colaboración en tareas de docencia universitaria no se limitó a los cursos regulares de las licenciaturas de química, física, biología y farmacia que atienden los profesores integrados en el Instituto, sino también a 2 cursos de postgrado y 6 de doctorado.

Los algo más de cien artículos publicados en revistas de difusión internacional por el personal científico del Instituto aparecieron, por regla general, en revistas de índice de impacto algo superior a 1.0. Entre esos artículos 16 se publicaron en las indicadas a continuación, con los índices de impacto respectivos: uno, en The Journal of the American Chemical Society, 5,65; dos, en Journal of Catalysis, 3,45; otros dos, en Chemistry of Materials, 3,27; cinco, en Physical Review $B$, 2,88; tres, en Langmuir, 2,85; uno, en Applied Catalysis B. Environment, 2,71; y dos, en Inorganic Chemistry, 2,74 .

En un futuro próximo el ICMS se propone reforzar su actividad en tres áreas de estudio de varios materiales: propiedades de superficie; síntesis y reactividad de muestras en polvo; y otras propiedades físicas. La incorporación al Centro de Isla de la Cartuja, junto con la homo- 
geneidad y carácter complementario de los proyectos y líneas de trabajo que se desarrollan en la nueva sede, permitirán abordar nuevas cuestiones en las áreas mencionadas y en otras áreas cercanas.

\section{Física de Materiales}

La UFM agrupa ahora a 18 personas: 17 profesores de la UPV (6 catedráticos, 8 titulares y 3 asociados) y un administrativo. Los profesores proceden de los departamentos de Física de Materiales, Física Aplicada, Física de la Materia Condensada, Electricidad y Electrónica, y Ciencias de la Computación e Inteligencia Artificial. Este núcleo inicial se irá ampliando de modo gradual por el CSIC, que creará un número de plazas equivalente. A continuación se relacionan media docena de líneas de trabajo actuales de la UFM que son expresivas de la naturaleza y la calidad de la labor que realiza.

Transferencia de energía y estados de carga de iones a velocidades lentas e intermedias, a plasmas y superficies. Con la teoría de muchos cuerpos se aborda la transferencia de energía de iones a plasmas asociados, con procesos de pérdida y captura de electrones por parte de los iones. También se interpretan recientes resultados experimentales de reflexión de iones lentos de helio en superficies de níquel, analizando la variación de la radiación emitida por salto entre niveles, así como el efecto vecindad en agregados moleculares y el efecto de la temperatura. De otra parte, en el nuevo campo de la interacción de antimateria con materia condensada, se estudiará el problema de la transferencia de energía de antiprotones lentos a un gas de electrones, tratando el apantallamiento no lineal dentro del método del funcional de la densidad.

Desarrollo de la interpretación teórica de la microscopía electrónica $y$ de efecto túnel. En el campo de la microscopía electrónica de transmisión se estudia cómo influyen la geometría de la muestra y su posición respecto al haz, en el espectro de pérdidas de energía de los electrones del haz; así como la dispersión, para el caso de blancos planos; y cómo influye también el soporte, en el espectro de pérdidas de energía correspondiente a pequeñas partículas. $\mathrm{Y}$ en el campo de la microscopía de efecto túnel, se estudia la influencia del tamaño de la punta y del potencial de la interfase así como los efectos locales de canje y correlación, en la medida experimental de la altura de la barrera.

Efectos dinámicos en el paso de iones altamente cargados por blancos sólidos. Con objeto de elaborar una teoría no lineal del proceso de transferencia de energía de iones a plasmas, se trata ahora de de- 
terminar el potencial inducido en blancos sólidos delgados cuando se bombardean con iones hidrógeno, a través de los cambios observados en las energías de unión del ión hidrógeno, teniendo en cuenta los efectos de la interacción espín-órbita y las correcciones radiativas.

Localización de electrones en superficies. Se estudian cuestiones como la vida media de electrones atrapados por superficies en estados imagen y cómo depende de la estructura cristalográfica y de la respuesta superficial del medio; los estados imágenes en materiales ferromagnéticos; el gas bidimensional de electrones; así como los efectos de superficie en el potencial de estela y en los electrones emitidos tras el paso de iones rápidos.

Estudio dinámico de la miscibilidad en aleaciones de polimeros. Usando técnicas de resonancia magnética nuclear de carbono-13 y de relajación dieléctrica, junto con técnicas clásicas, se estudian varios sistemas con un componente común, PVME, para conocer mejor tanto la dinámica de aleaciones de polímeros amorfos como el fenómeno de la separación de fases.

Estudio de la dinámica de polímeros constituyentes de vidrios, en la región de coalescencia de las relajaciones alfa y beta. Se trata de clarificar los mecanismos moleculares que controlan la coalescencia de las relajaciones alfa y beta mediante la aplicación de varias técnicas experimentales: la resonancia magnética nuclear, la dispersión cuasielástica de neutrones y, especialmente, las técnicas de espectroscopía dieléctrica, que permiten actualmente estudiar frecuencias útiles en polímeros hasta $10^{9} \mathrm{~Hz}$ y por tanto no sólo la región de la coalescencia, sino también, eligiendo los polímeros adecuados, la relajación alfa-beta.

\section{El futuro}

Si bien el futuro llega siempre antes de lo previsto porque... ha comenzado ya... está ahí, al alcance de la mano, quizás quepan ahora unas consideraciones finales sobre el porvenir que espera a la ciencia y tecnología de materiales en general, y en el ámbito del CSIC en particular. Alguna vez se ha dicho que todas las previsiones sobre el futuro de la ciencia han tenido como característica común la constancia en el error. Aunque esto encierra parte de verdad, pues los saberes experimentales dan muchas sorpresas, vale la pena correr una vez más el riesgo, para afirmar en primer término y sin lugar a dudas, que la ciencia y la tecnología de materiales tienen futuro, un futuro asegurado. Por muchas razones. 
Está, en primer término, el afán de saber, sin el cual no habría conocimiento nuevo alguno. Está, también, lo mucho que queda por conocer: el mundo material, todo lo que nos rodea, es objeto de observación y... caja continua de sorpresas. A pesar de que los científicos usan la imaginación, la realidad logra siempre superarla. El futuro será mucho más sorprendente de lo que quepa imaginar ahora. Está de otra parte, por lo que atañe al área de CTM, que satisface aspiraciones generalizadas de las gentes de nuestro tiempo: bienestar material, calidad de vida, ambiente más sano... Y, por último, está la actitud que adopten quienes destinan los recursos dinerarios, y las personas que cultivan estos saberes.

A los políticos de este país se les ha repetido una y otra vez que el tanto por mil -porque no llega al uno por ciento- del producto nacional bruto que dedican a $\mathrm{I}+\mathrm{D}$ es muy inferior al de países comparables; y que para tener buenos resultados en ciencias experimentales hace falta una masa crítica que aún no hemos logrado en España. Es decir, que trescientos científicos dedicados a CTM en el CSIC son pocas personas. Es preciso ser optimistas y pensar que, por hacer realidad los deseos de sus votantes, los políticos acabarán aumentando razonablemente ese tanto por mil y ese número de científicos.

En cuanto a los investigadores del área de CTM, es verdad que pocos han tomado la ciencia o la tecnología de materiales como fin último de sus propias vidas. La mayor parte buscan la fuente de inspiración a que se refiere el lema de este artículo, en algo que consideran superior: Dios, los demás, el progreso, o el propio yo. Pero la inmensa mayoría dedican a la CTM más tiempo del que establecen las normas laborales y desempeñan su trabajo con entusiasmo. Entusiasmo que es aún más vivo entre los jóvenes, en particular los doctores que aún no han logrado una plaza permanente.

Hace pocos años, desajustes entre el número de becas postdoctorales previstas y el de plazas que podría absorber el sistema español de $\mathrm{I}+\mathrm{D}$, dejaron en el camino a buen número de doctores bien formados. Gran error. La reacción de la Administración a sus protestas encontró el año pasado una solución de parte del problema, que disminuyó apreciablemente el número de los que tuvieron que renunciar a la profesión investigadora. En todo caso, la mayor parte de quienes la inician ahora en nuestro país son laboriosos, activos, apasionados. Parece como si hubieran escuchado el grito de Pávlov a los jóvenes investigadores de su tiempo:

¿Qué puedo desear para la juventud de mi país que se dedica a la ciencia?... pasión. Recordad que la ciencia exige toda la vida de 
un hombre. Ni siquiera con dos vidas sería suficiente. Apasionaos por vuestro trabajo y por vuestra investigación.

Por todas estas razones se puede augurar futuro, buen futuro, al área de CTM en nuestro país. Con el paso del tiempo habrá, sin duda, resultados mayores y mejores. Aunque tenía razón Pushkin cuando afirmaba que la inspiración es tan necesaria para la geometría como para la poesía ¿por qué no va a haber aquí buenos científicos, si hemos tenido siempre y tenemos ahora tan buenos poetas?

\section{Agradecimientos}

Este trabajo debe mucho a la colaboración de buen número de personas: María Jesús Almeida, Mar Caso, Esperanza Iglesias y Matilde Villaroig, de las bibliotecas del ICMM, general de Medinaceli, del Instituto de Química Física Rocasolano y del Instituto de Geografía; los directores de los centros, Profs. Pablo Javier Alonso, María del Carmen Andrade, Angel Caballero, Pedro M. Echenique, Antonio Formoso, María del Carmen Mijangos, Carlos Miravitlles, Agustín Rodríguez, Federico Soria; y, por descontado, los Profs. Enric Canadell, coordinador del área de CTM, y su antecesor, J.C. Serna. Y otro tanto debe a la del Dr. Francisco J. Valle, Vicedirector del ICV, y a las de D. Tomás Fraile, Subdirector General de Actuación Económica del CSIC, D. José Antonio Alcaín, Jefe del Servicio de Presupuestos, también del CSIC, y Dña. Josefa Rodríguez, Gerente del ICMM. Para todos, mi agradecimiento más vivo.

\section{Acrónimos más empleados}

$\begin{array}{ll}\text { AMIET } & \text { Asociación de Miembros del Instituto Eduardo Torroja } \\ \text { CAM } & \text { Comunidad Autónoma de Madrid } \\ \text { CENIM } & \text { Centro Nacional de Investigaciones Metalúrgicas } \\ \text { CICYT } & \text { Comisión Interministerial de Ciencia y Tecnología } \\ \text { CIETAN } & \begin{array}{l}\text { Conformidad del Instituto Eduardo Torroja. Asociación Na- } \\ \text { cional. }\end{array} \\ \text { COST } & \begin{array}{l}\text { European Cooperation in the field of Scientific and Technical } \\ \text { Research }\end{array} \\ \text { CNRS } & \begin{array}{l}\text { Centre National de la Récherche Scientifique } \\ \text { CSIC }\end{array} \\ \text { CTM Consejo Superior de Investigaciones Científicas } \\ \text { Ciencia y Tecnología de Materiales }\end{array}$




\section{Isidoro Rasines}

CVD Chemical Vapor Deposition

DIT Documento de Idoneidad Técnica

DQV Deposición química a partir de vapor

ICMA Instituto de Ciencia de Materiales de Aragón

ICMB Instituto de Ciencia de Materiales de Barcelona

ICMM Instituto de Ciencia de Materiales de Madrid

ICMS Instituto de Ciencia de Materiales de Sevilla

ICTP Instituto de Ciencia y Tecnología de Polímeros

ICV Instituto de Cerámica y Vidrio

I+D Investigación y Desarrollo

IERS Instalación Europea de Radiación de Sincrotrón, Grenoble

IETCC Instituto Eduardo Torroja de Ciencias de la Construcción

ILL Instituto Laue Langevin, Grenoble

IR Infrarrojo

ISI Institute for Scientific Information, Filadelfia

LEA Laboratorio Europeo Asociado

LURE Laboratorio para el Uso de Radiación Electromagnética, Orsay

OTAN Organización del Tratado del Atlántico Norte

UE Unión Europea

UFM Unidad de Física de Materiales

UZ Universidad de Zaragoza

\section{Referencias}

1 S. DE AZA, con la colaboración de J.C. SERNA y V. ORERA. Áreas de investigación, en El CSIC. Medio Siglo de Investigación. R. Pastor y A. Sánchez Álvarez-Insúa, coordinadores. Madrid, 1996, págs. 61 y 115-125.

2 Centro para el Desarrollo Tecnológico Industrial. Ministerio de Industria y Energía. Materiales Avanzados. Madrid, 1994, pág. 111.

3 Science Citation Index. Journal Citation Reports. Institute for Scientific Information. Philadelphia.

4 CSIC. Plan de Actuación 1995-1999. Madrid, 1995. págs. 78-85. 


\section{Ciencia y Tecnología de materiales}

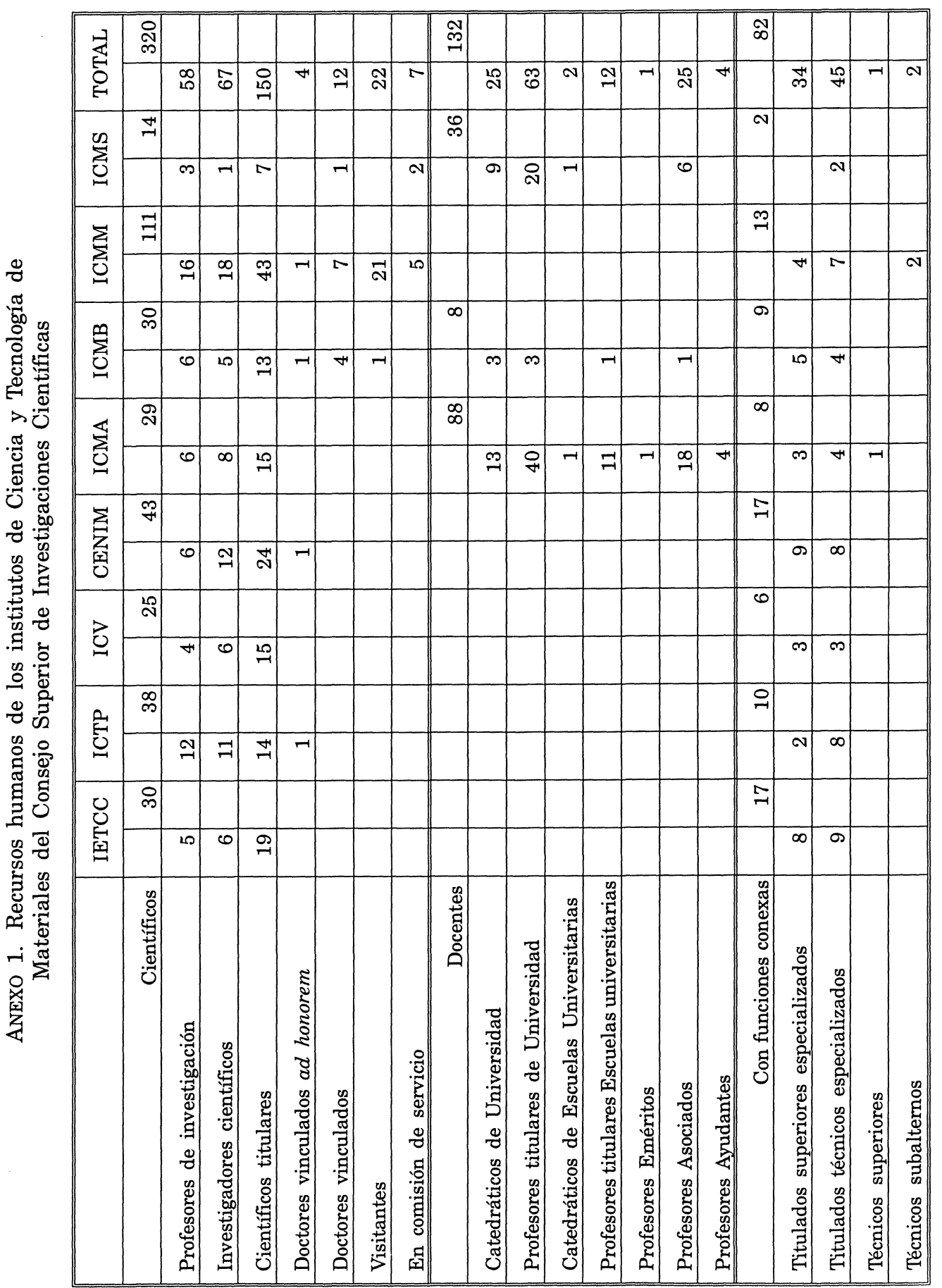




\section{Isidoro Rasines}

\begin{tabular}{|c|c|c|c|c|c|c|c|c|c|c|c|c|c|c|c|c|c|c|c|c|}
\hline \multirow{2}{*}{ 悹 } & \multicolumn{2}{|l|}{$\begin{array}{c}\infty \\
\\
\end{array}$} & & & if & & & & 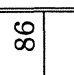 & & \multirow{2}{*}{$\sigma$} & \multirow{2}{*}{$\stackrel{\infty}{-1}$} & \multirow{2}{*}{$\sigma$} & \multirow{2}{*}{ จ } & \multirow{2}{*}{7} & \multicolumn{2}{|l|}{$\begin{array}{l}8 \\
0 \\
-1\end{array}$} & & \multirow{2}{*}{$\mathscr{1}$} \\
\hline & & is & $\begin{array}{l}0 \\
0\end{array}$ & $\stackrel{20}{-10}$ & & 吕 & 9 & 20 & & -1 & $\stackrel{\infty}{-\infty}$ & & & & & & & $\mathfrak{N}$ & $\mathfrak{N}$ & \\
\hline \multirow{2}{*}{$\sum_{0}^{\pi}$} & 7 & & & & +4 & & & & 20 & & & & & & & & $\sigma$ & & & \\
\hline & & & 7 & & & 7 & $\infty$ & & & & 7 & $\pi$ & & 7 & $N$ & & & 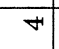 & 10 & \\
\hline \multirow{2}{*}{ 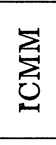 } & $\stackrel{0}{-1}$ & & & & + & & & & $\sim$ & & & & & & & & 8 & & & \\
\hline & & 0 & 어 & & & -1 & $\infty$ & & & & $\infty$ & 7 & $\mathrm{~N}$ & & & 7 & & $\stackrel{0}{\rightarrow}$ & $\stackrel{\infty}{\sim}$ & $\stackrel{1}{\pi}$ \\
\hline \multirow{2}{*}{$\sum_{0}^{\infty}$} & + & & & & 20 & & & & + & & & & & & & & ล) & & & \\
\hline & & & $\infty$ & -1 & & $\mathbf{N}$ & $\infty$ & & & & & 7 & 7 & -1 & 7 & & & 7 & $\infty$ & \\
\hline \multirow{3}{*}{$\sum_{0}^{\mathbb{Z}}$} & 0 & & & & $\mathbf{N}$ & & & & & & & & & & & & $=$ & & & \\
\hline & & & & & & & & & & & & & & & & & & & & \\
\hline & & & 0 & & & -1 & -1 & & & & & & & & & & & $\stackrel{20}{-1}$ & $N$ & \\
\hline \multirow{2}{*}{ 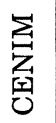 } & o & & & & 7 & & & & $\underset{\sim}{\sim}$ & & & & & & & & $\pi$ & & & \\
\hline & & $\mathscr{\infty}$ & $\mathscr{\infty}$ & $\pi$ & & $\infty$ & & $\infty$ & & & 7 & -1 & -1 & $\infty$ & 20 & 7 & & $\nabla$ & $\infty$ & \\
\hline \multirow{2}{*}{ 己 } & 番 & & & & $\infty$ & & & & $\mathbb{N}$ & & & & & & & & $\mathbb{N}$ & & & \\
\hline & & का & $\mathscr{m}$ & $\bar{N}$ & & $N$ & 7 & & & & 10 & & 0 & 7 & 20 & 20 & & $N$ & & \\
\hline \multirow{2}{*}{ 号 } & $\stackrel{\infty}{\sim}$ & & & & 0 & & & & + & & & & & & & & $\sigma$ & & & \\
\hline & & 0 & 7 & -1 & & $m$ & 7 & $N$ & & & & $\infty$ & 7 & & & & & $\pi$ & 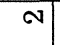 & \\
\hline \multirow{2}{*}{$\begin{array}{l}\text { O } \\
\text { 置 }\end{array}$} & $\mathscr{\infty}$ & & & & 0 & & & & న్లు & & & & & & & & 金 & & & \\
\hline & & 0 & 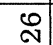 & $\pi$ & & $\boldsymbol{I}$ & $\pi$ & & & 7 & $\infty$ & $N$ & - & $\infty$ & $\pi$ & $\nabla$ & & 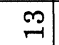 & $\stackrel{\mathbb{N}}{\mathrm{N}}$ & \\
\hline & 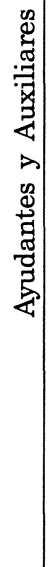 & 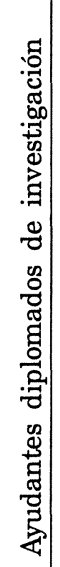 & 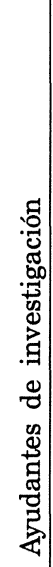 & 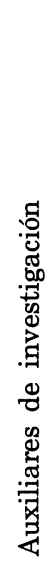 & 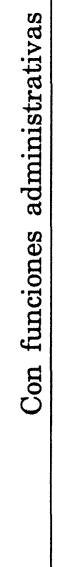 & $\begin{array}{c} \\
\\
\\
0\end{array}$ & 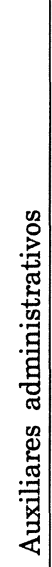 & 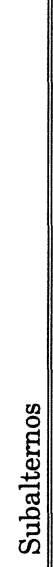 & 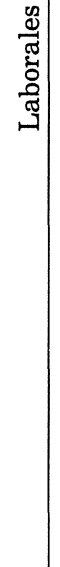 & 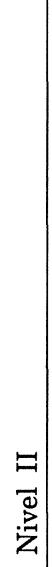 & $\begin{array}{l}\exists \\
\vec{d} \\
\overrightarrow{3} \\
\vec{z}\end{array}$ & $\begin{array}{l}Z \\
\vec{d} \\
\stackrel{\vec{c}}{z}\end{array}$ & 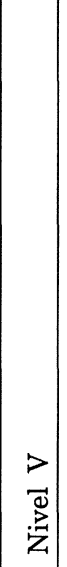 & $\begin{array}{l}5 \\
\vec{d} \\
\vec{z} \\
\dot{z}\end{array}$ & 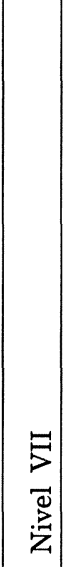 & 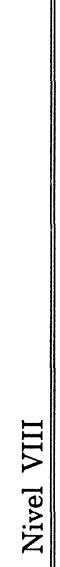 & 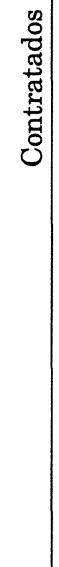 & 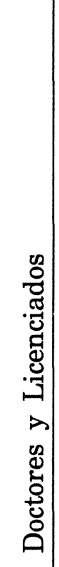 & 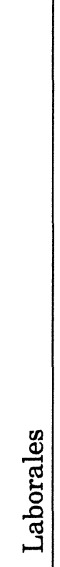 & : \\
\hline
\end{tabular}




\section{Ciencia y Tecnología de materiales}

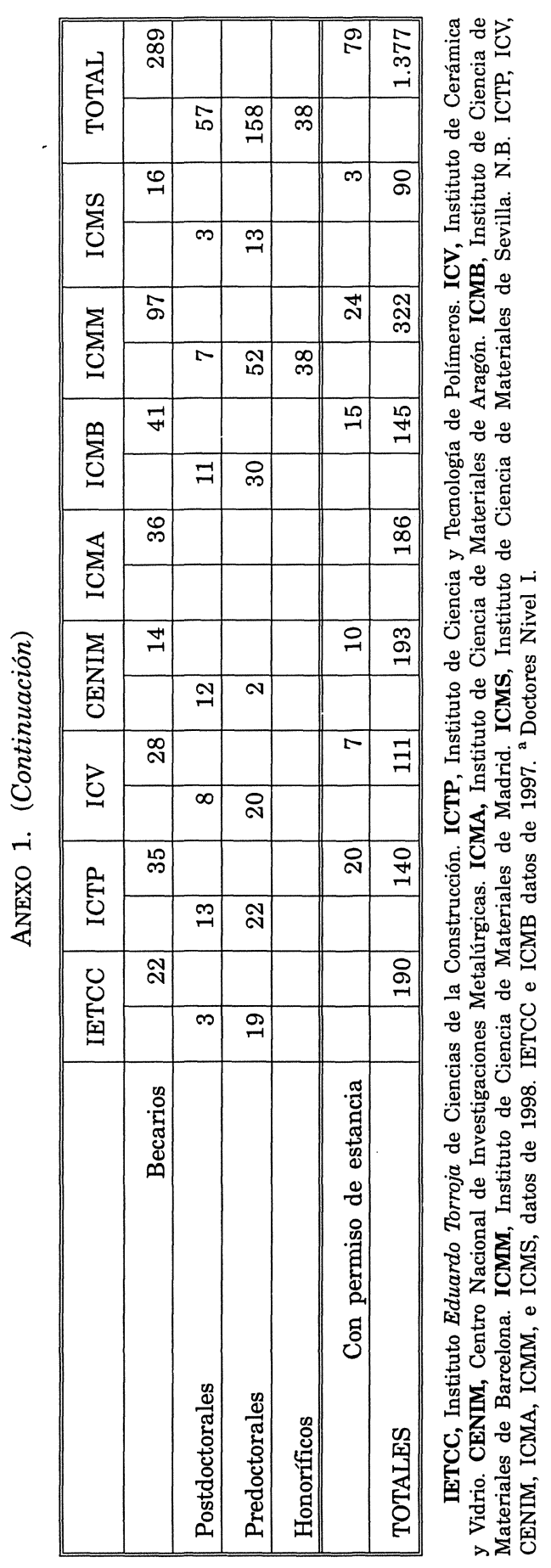




\section{Ciencia y Tecnología de materiales}

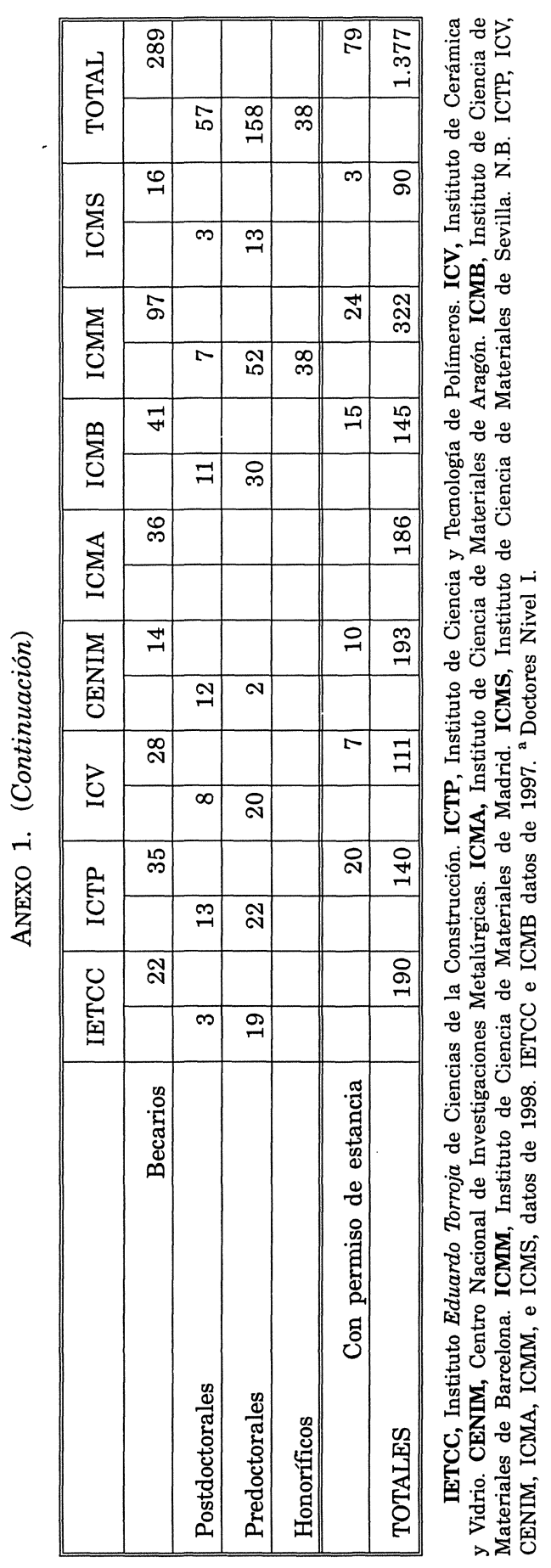




\section{Isidoro Rasines}

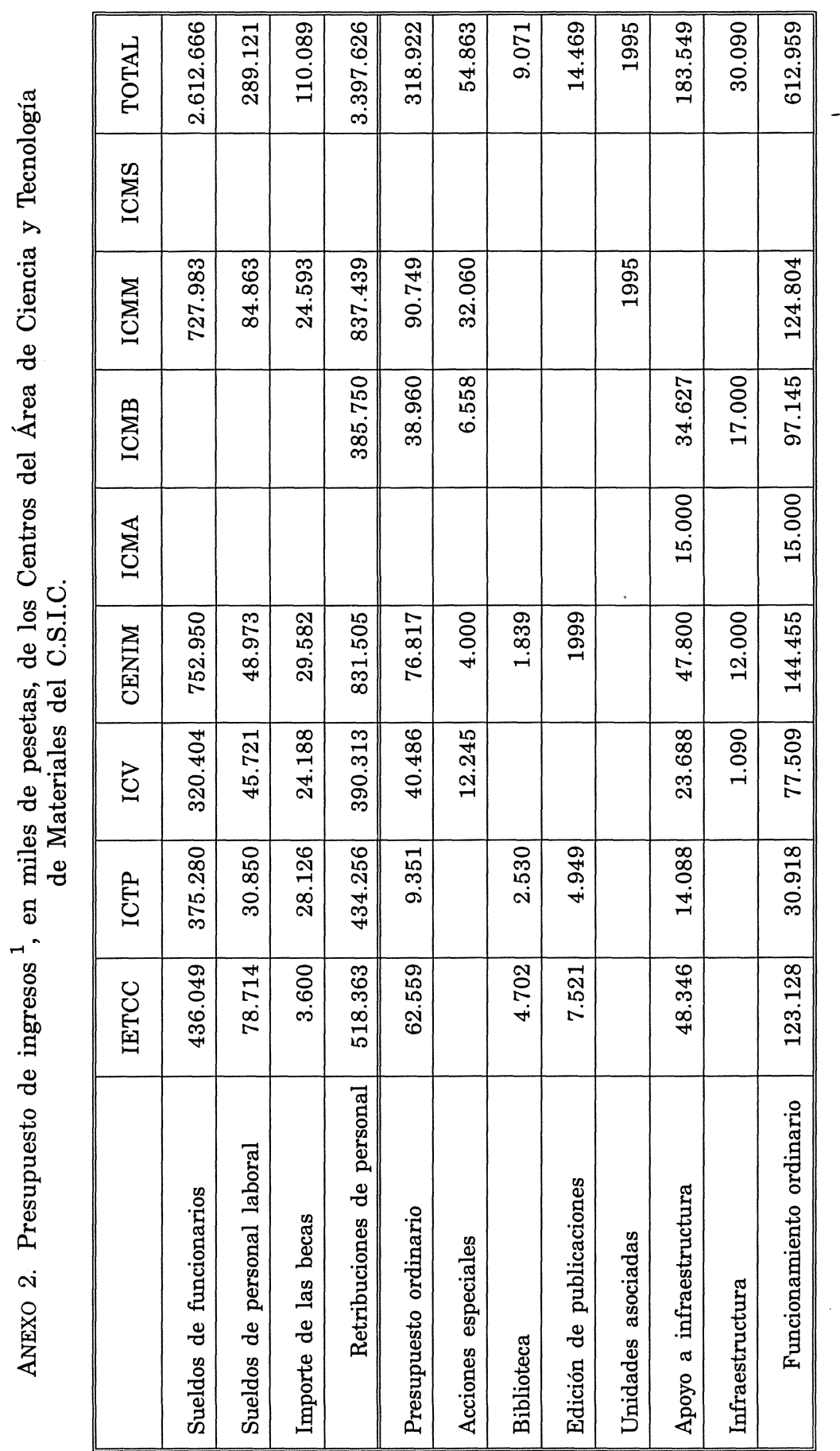




\section{Isidoro Rasines}

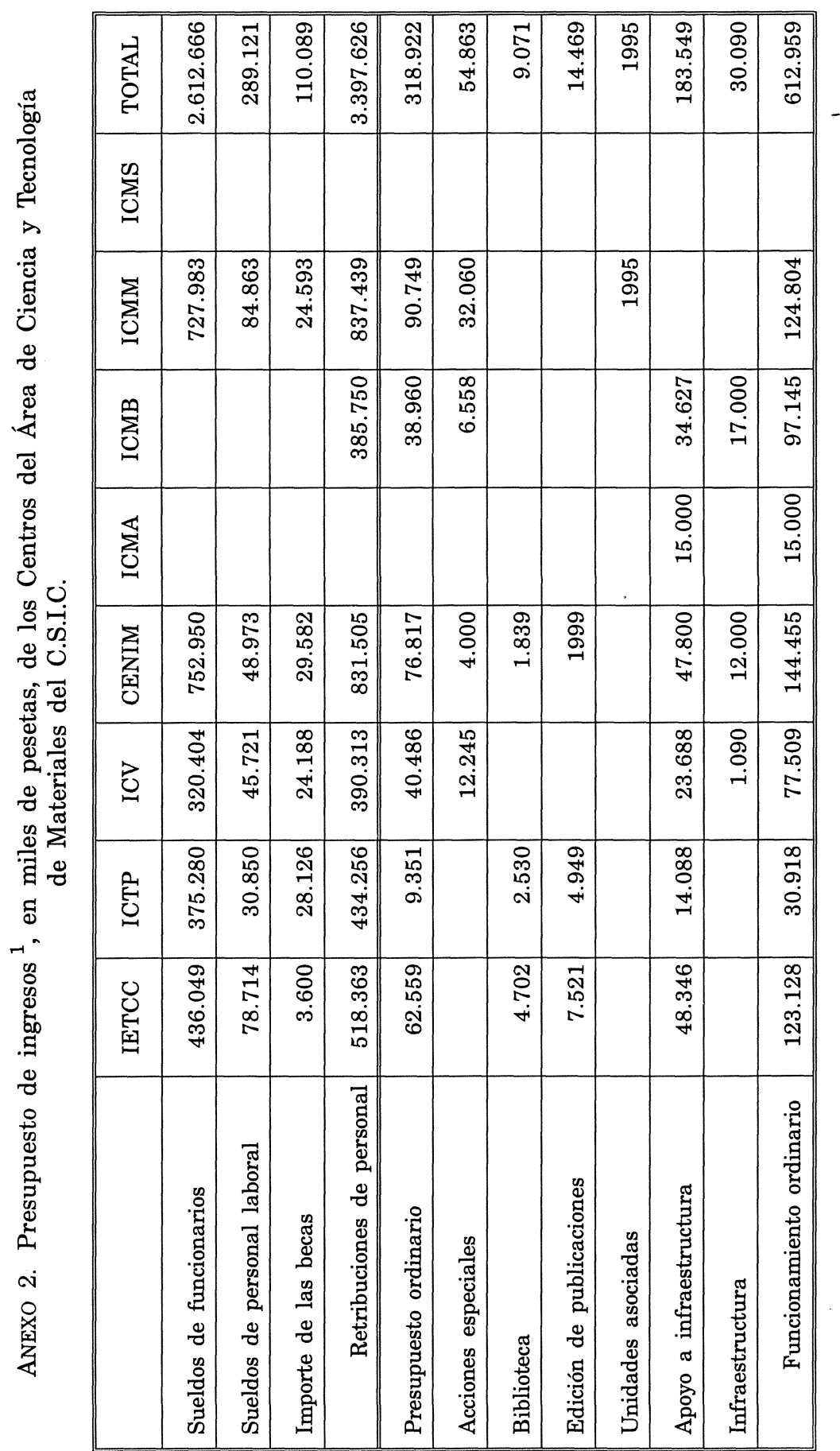




\section{Ciencia y Tecnología de materiales}

\begin{tabular}{|c|c|c|c|c|c|c|c|c|c|c|c|}
\hline 㞾 & 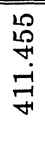 & 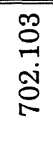 & $\begin{array}{l}\frac{8}{4} \\
-1 \\
0 \\
0\end{array}$ & 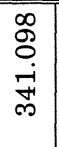 & 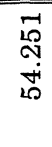 & 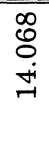 & 离 & $\begin{array}{l}\infty \\
\infty \\
\infty \\
\infty \\
0\end{array}$ & 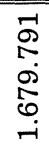 & 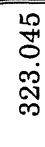 & 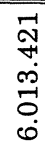 \\
\hline$\sum_{0}^{\mathbb{N}}$ & 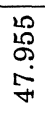 & 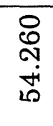 & 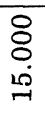 & & & & & $\begin{array}{l}8 \\
\& \\
\infty \\
7 \\
7\end{array}$ & 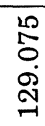 & & 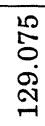 \\
\hline$\sum_{0}^{\sum_{0}}$ & 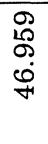 & 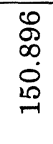 & 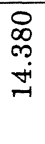 & $\begin{array}{l}\vec{N} \\
\text { N } \\
\text { N }\end{array}$ & & & & $\begin{array}{l}10 \\
\infty \\
\infty \\
0 \\
0\end{array}$ & $\begin{array}{l}\vec{F} \\
\text { on } \\
\stackrel{\text { N }}{\text { N }}\end{array}$ & $\begin{array}{l}\vec{\infty} \\
\text { 今 } \\
\text { సิ }\end{array}$ & $\begin{array}{l}\stackrel{20}{0} \\
0 \\
0 \\
-9 \\
-1\end{array}$ \\
\hline 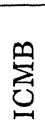 & 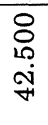 & 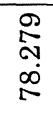 & $\begin{array}{l}8 \\
\stackrel{8}{\circ} \\
\stackrel{-1}{-1}\end{array}$ & \begin{tabular}{l}
8 \\
8 \\
20 \\
\multirow{4}{*}{} \\
\end{tabular} & & & & & $\begin{array}{l}0 \\
\mathbb{0} \\
\text { N } \\
\infty \\
\infty \\
-1\end{array}$ & & $\begin{array}{l}\text { J } \\
-1 \\
-1 \\
\text { - }\end{array}$ \\
\hline 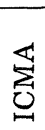 & 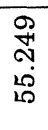 & 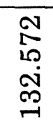 & 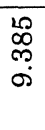 & $\begin{array}{l}0 \\
0 \\
0 \\
0\end{array}$ & & 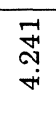 & & $\begin{array}{l}\mathbb{W} \\
\mathbb{d} \\
\infty \\
\infty\end{array}$ & 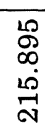 & & 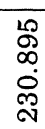 \\
\hline 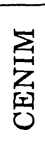 & 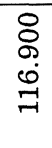 & \begin{tabular}{l}
0 \\
\multirow{1}{1}{} \\
0 \\
0
\end{tabular} & 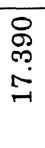 & 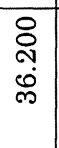 & $\begin{array}{l}\stackrel{8}{\circ} \\
\stackrel{\infty}{r} \\
\end{array}$ & 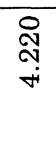 & & $\begin{array}{l}\stackrel{0}{\infty} \\
\text { o } \\
\infty \\
\infty\end{array}$ & $\begin{array}{l}8 \\
8 \\
\stackrel{1}{1} \\
\text { ล }\end{array}$ & $\begin{array}{l}\text { 尺े } \\
\stackrel{0}{0} \\
\stackrel{0}{0}\end{array}$ & 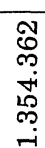 \\
\hline 己己 & 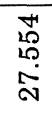 & 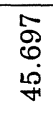 & & 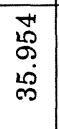 & 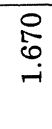 & 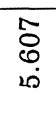 & & & 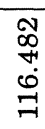 & $\begin{array}{l}\tilde{0} \\
\varnothing \\
\infty \\
\infty\end{array}$ & $\begin{array}{l}\mathscr{0} \\
0 \\
0 \\
0 \\
0\end{array}$ \\
\hline 苞 & 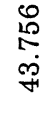 & 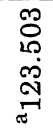 & & $\begin{array}{l}\text { Ñ } \\
\text { ô. } \\
\stackrel{8}{8}\end{array}$ & & & & 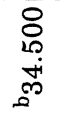 & 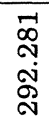 & & 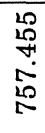 \\
\hline $\begin{array}{l}\text { O } \\
\text { 鼠 }\end{array}$ & 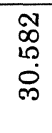 & $\begin{array}{l}\mathscr{0} \\
7 \\
7 \\
0\end{array}$ & & 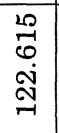 & $\begin{array}{l}\vec{\infty} \\
\stackrel{0}{0} \\
i\end{array}$ & & $\underset{\substack{+1 \\
\hdashline}}{ }$ & & $\begin{array}{l}\infty \\
\stackrel{\infty}{\circ} \\
\stackrel{+}{+} \\
\text { N }\end{array}$ & & $\begin{array}{l}\text { के } \\
+ \\
\stackrel{0}{0} \\
\infty \\
\infty\end{array}$ \\
\hline & 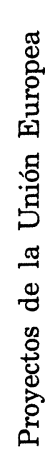 & 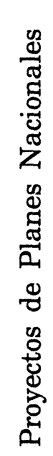 & 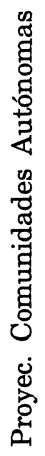 & 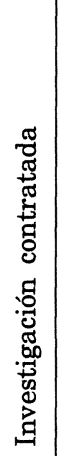 & 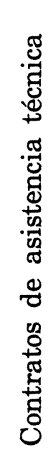 & 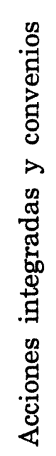 & 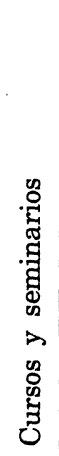 & $\begin{array}{l}0 \\
\tilde{g}_{0}^{\circ}\end{array}$ & 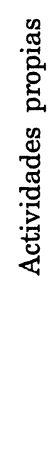 & 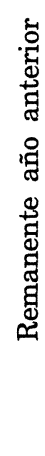 & $\begin{array}{l}\text { 吕 } \\
\text { 离 } \\
\text { 总 }\end{array}$ \\
\hline
\end{tabular}

